\title{
VERY STRONG EMISSION-LINE GALAXIES IN THE WFC3 INFRARED SPECTROSCOPIC PARALLEL SURVEY AND IMPLICATIONS FOR HIGH-REDSHIFT GALAXIES*, ${ }^{*}$
}

\author{
H. Atek ${ }^{1}$, B. Siana ${ }^{2}$, C. Scarlata ${ }^{3}$, M. Malkan ${ }^{4}$, P. McCarthy $^{5}$, H. Teplitz ${ }^{6}$, A. Henry ${ }^{7}$, J. Colbert ${ }^{1}$, C. Bridge $^{2}$, \\ A. J. Bunker ${ }^{8}$, A. Dressler ${ }^{5}$, R. A. E. Fosbury ${ }^{9}$, N. P. $\mathrm{Hathi}^{5}$, C. MarTin ${ }^{7}$, N. R. Ross ${ }^{4}$, And H. Shim ${ }^{1}$ \\ ${ }^{1}$ Spitzer Science Center, Caltech, Pasadena, CA 91125, USA \\ 2 Department of Astronomy, Caltech, Pasadena, CA 91125, USA \\ ${ }^{3}$ Department of Astronomy, University of Minnesota-Twin Cities, Minneapolis, MN 55455, USA \\ ${ }^{4}$ Department of Physics and Astronomy, University of California, Los Angeles, CA, USA \\ ${ }^{5}$ Observatories of the Carnegie Institution for Science, Pasadena, CA 91101, USA \\ ${ }^{6}$ Infrared Processing and Analysis Center, Caltech, Pasadena, CA 91125, USA \\ ${ }^{7}$ Department of Physics, University of California, Santa Barbara, CA 93106, USA \\ ${ }^{8}$ Department of Physics, University of Oxford, Denys Wilkinson Building, Keble Road, Oxford OX1 3RH, UK \\ ${ }^{9}$ Space Telescope-European Coordinating Facility, Garching bei München, Germany \\ Received 2011 July 8; accepted 2011 September 3; published 2011 November 29
}

\begin{abstract}
The WFC3 Infrared Spectroscopic Parallel Survey uses the Hubble Space Telescope (HST) infrared grism capabilities to obtain slitless spectra of thousands of galaxies over a wide redshift range including the peak of star formation history of the universe. We select a population of very strong emission-line galaxies with rest-frame equivalent widths (EWs) higher than $200 \AA$. A total of 176 objects are found over the redshift range $0.35<z<2.3$ in the $180 \mathrm{arcmin}^{2}$ area that we have analyzed so far. This population consists of young and low-mass starbursts with high specific star formation rates (sSFR). After spectroscopic follow-up of one of these galaxies with Keck/Low Resolution Imaging Spectrometer, we report the detection at $z=0.7$ of an extremely metal-poor galaxy with $12+$ $\log (\mathrm{O} / \mathrm{H})=7.47 \pm 0.11$. After estimating the active galactic nucleus fraction in the sample, we show that the high-EW galaxies have higher sSFR than normal star-forming galaxies at any redshift. We find that the nebular emission lines can substantially affect the total broadband flux density with a median brightening of $0.3 \mathrm{mag}$, with some examples of line contamination producing brightening of up to 1 mag. We show that the presence of strong emission lines in low- $z$ galaxies can mimic the color-selection criteria used in the $z \sim 8$ dropout surveys. In order to effectively remove low-redshift interlopers, deep optical imaging is needed, at least 1 mag deeper than the bands in which the objects are detected. Without deep optical data, most of the interlopers cannot be ruled out in the wide shallow $H S T$ imaging surveys. Finally, we empirically demonstrate that strong nebular lines can lead to an overestimation of the mass and the age of galaxies derived from fitting of their spectral energy distribution (SED). Without removing emission lines, the age and the stellar mass estimates are overestimated by a factor of 2 on average and up to a factor of 10 for the high-EW galaxies. Therefore, the contribution of emission lines should be systematically taken into account in SED fitting of star-forming galaxies at all redshifts.
\end{abstract}

Key words: cosmology: observations - galaxies: evolution - galaxies: high-redshift - galaxies: statistics infrared: galaxies - surveys

Online-only material: color figures

\section{INTRODUCTION}

The characterization of galaxies undergoing their first major star formation episode is of great interest in modern astrophysics. This class of galaxies is expected to host young and massive stars ionizing the interstellar medium, and therefore to exhibit strong nebular emission lines. However, many efforts dedicated to the understanding of galaxy formation and evolution are in general limited by different factors or colored by the way in which galaxy samples are assembled. Broadband surveys yield magnitude-limited samples that are biased toward bright continuum objects such as the Lyman break galaxies (LBGs; Steidel et al. 1996; Shapley et al. 2003; Vanzella et al.

\footnotetext{
* Based on observations made with the NASA/ESA Hubble Space Telescope, which is operated by the Association of Universities for Research in Astronomy, Inc., under NASA contract NAS 5-26555. These observations are associated with programs 11696 and 12283.

$\dagger$ Some of the data presented herein were obtained at the W. M. Keck Observatory, which is operated as a scientific partnership among the California Institute of Technology, the University of California, and the National Aeronautics and Space Administration. The Observatory was made possible by the generous financial support of the W. M. Keck Foundation.
}

2009; Hathi et al. 2010), and can favor relatively massive galaxy populations. On the other hand, ground-based searches for restframe optical emission lines from high-redshift galaxies are severely impacted by the bright NIR background. Observing in small wavelength windows between $\mathrm{OH}$ terrestrial airglow will significantly restrict the survey volume.

In this context, space-based observations offer considerable advantages for the detection of $z \sim 1-2$ emission-line galaxies. The Wide field Camera 3 (WFC3) onboard the Hubble Space Telescope (HST) has dramatically improved the power of slitless spectroscopy from above the atmosphere. Beginning in cycle 17, we are conducting the WFC3 Infrared Spectroscopic Parallel (WISP) Survey. In about 500 orbits of parallel observations, the program will obtain slitless spectra of galaxies at the peak of the star formation history (SFH) of the universe (e.g., Hopkins 2004). The $G_{102}$ and $G_{141}$ IR grisms offer continuous wavelength coverage from 0.8 to $1.7 \mu \mathrm{m}$, allowing the selection of emissionline galaxies at $0.35<z<2.5$, and potentially bright $\mathrm{Ly} \alpha$ emitters at $z>6.5$ (see Atek et al. 2010, for a complete description of the survey). 
In this paper, we present a sample of emission-line objects with extremely high equivalent widths (EWs). The EW of recombination lines indicates the ratio of the current star formation rate (SFR) to the past average SFR of a galaxy (e.g., Kennicutt 1998). Therefore, the high-EW selection picks up galaxies with a strong ongoing star formation episode.

Previously, ground-based narrowband surveys were used to identify a large sample of ultra-strong emission-line galaxies (USELs; Kakazu et al. 2007; Hu et al. 2009), but were restricted to a narrow range of redshifts up to $z=1$. In the local universe, Cardamone et al. (2009) found in the Sloan Digital Sky Survey (SDSS) compact star-forming galaxies, called "green peas," that have very blue $r-i$ colors because of their very strong [O III] $\lambda 5007$ emission line. Thanks to the unprecedented sensitivity of the WFC3/IR detectors, the depth of the direct imaging combined with the resolution and the wavelength coverage of the grism spectroscopy allows us to uncover this particular class of objects independently of the continuum brightness over a wide redshift range.

The selection of high-EW star-forming galaxies with the WFC3 grisms probes a lower mass range than previous studies at $z>1$ (e.g., Erb et al. 2006b; Daddi et al. 2007). This allows us to compare the star formation efficiency of these dwarf galaxies with normal star-forming galaxies at $z \sim 1-2$. Indeed, several studies have established a correlation between the SFR and the stellar mass up to redshift $z=7$ (e.g., Brinchmann \& Ellis 2000; Elbaz et al. 2007; Noeske et al. 2007b; Daddi et al. 2007; Pannella et al. 2009; Oliver et al. 2010; Labbé et al. 2010). The shape and evolution of this relationship are interpreted as the result of smooth gas accretion (Bouché et al. 2010; Davé et al. 2011). However, at high redshifts, the studies focused on a specific mass range due to the difficulty of selecting faintcontinuum galaxies.

In a broader context, we also discuss the implications of these strong line emitters on the selection of $z \sim 8$ galaxies and on the physical properties of galaxies derived from spectral energy distribution (SED) modeling.

We present in Section 2 the WISP Survey observations and the data reduction. Section 3 is devoted to the followup observations. The selection of the high-EW population is presented in Section 4. In Section 6, we discuss the mass and star formation properties of the high-EW galaxies. We present in Section 7 the metallicity measurement of one example and put it into context. We investigate the contribution of nebular lines to the total broadband flux density and their astrophysical implications in Sections 5 and 5.2. Our conclusions are given in Section 8 . Throughout, we assume a $\Lambda$-dominated flat universe, with $H_{0}=70 \mathrm{~km} \mathrm{~s}^{-1} \mathrm{Mpc}^{-1}, \Omega_{\Lambda}=0.7$, and $\Omega_{m}=0.3$. All magnitudes are in the $\mathrm{AB}$ system.

\section{THE WISP SURVEY}

The WISP Survey exploits the powerful pure parallel mode of the HST to obtain slitless spectra of nearly 200 uncorrelated fields in 500 orbits within two observing programs (PI: M. Malkan): GO 11696 and 12283 (Infrared Survey of Star Formation Across Cosmic Time). During long integrations with the Cosmic Origins Spectrograph (COS; Osterman et al. 2011) or the Space Telescope Imager and Spectrograph (STIS; Woodgate et al. 1998), the WISP program uses WFC3 (Kimble et al. 2008) to observe an adjacent field in parallel, at a fixed offset of about $5^{\prime}$ from the primary target. The data include slitless spectroscopy with $G_{102}(0.8-1.2 \mu \mathrm{m})$ and $G_{141}(1.1-1.7 \mu \mathrm{m})$ grisms, and direct NIR imaging with the F110W and F140W filters. As of 2010 August, we added the F475X and F600LP filters of the WFC3/UVIS channel to our observing program, with typical exposure times of $400 \mathrm{~s}$ in each band. The fields analyzed in this paper do not have UVIS observations. For the long visits (typically four orbits or more), we observe with both grisms with a typical exposure time ratio of $G_{102}: G_{141}=2.5: 1$. For shorter visits, the fields are observed in $G_{141}$ only. The direct images are obtained in the same orbits as the grism imaging with a $\sim 6: 1$ grism:direct integration time ratio. It is necessary for the spectral extraction from slitless data to use the direct images to provide the input catalog of objects. The object positions, sizes, and shapes are then used, in conjunction with a dispersion solution that translates the position from the direct image to the spectral trace in the grism one, to extract flux and wavelength calibrated two-dimensional and one-dimensional spectra.

In this paper, we analyzed a total of 54 fields presented in Table 1, among which two have optical follow-up (cf. Section 3). We note that our effective area represents about $70 \%$ of the full WFC3/IR frame because of two things. First, objects that fall outside the right edge of the direct image frame will have their zero-order spectra falling in the right side of the grism frame (zeroth order is dispersed to the left side of the object position). Thus, we cannot correct the zeroth-order contamination in that part of the detector. Second, the left edge of the grism frame will receive spectra from objects that are outside the direct image (first order dispersed to the right side of the object position), preventing any flux or wavelength calibration for those sources.

All the data were processed with the WFC3 pipeline CALWF3 (ver. 2.1) to correct for bias, dark, flat-field, and gain variations. Then, the slitless extraction package aXe 2.0 (Kümmel et al. 2009) is used for the spectral extraction. Spectra with important contamination are removed from the analysis because the contamination estimate of aXe is not sufficiently accurate. A complete description of the data reduction steps is presented in Atek et al. (2010).

\section{OPTICAL FOLLOW-UP SPECTROSCOPY AND IMAGING}

We have obtained optical spectra of objects in two adjacent WISP fields with the Low Resolution Imaging Spectrometer (LRIS; Oke et al. 1995; Steidel et al. 2004) on the Keck I telescope. The two WFC3 fields, WISP5 and WISP7 in Table 1, are immediately adjacent to each other and fit within one LRIS slit mask. We assigned 1".2 wide slits to 13 emissionline objects, five of which belong to the high-EW sample. Three exposures of $1800 \mathrm{~s}$ each were used for a total integration of $5400 \mathrm{~s}$. The $4001 \mathrm{~mm}^{-1}$ grism blazed at $3400 \AA$ was used on the blue arm and the $6001 \mathrm{~mm}^{-1}$ grating blazed at $1.0 \mu \mathrm{m}$ was used on the red arm for a pixel scale of $\sim 1.1 \AA$ pixel $^{-1}$ and $\sim 0.8 \AA \mathrm{pixel}^{-1}$, respectively. The plate scale is $0^{\prime \prime} 135 \mathrm{pixel}^{-1}$ and the spectral resolution for an object that fills the slit is about $8.1 \AA$ and $5.6 \AA$ in the blue and red side, respectively. The seeing during observations was around $1^{\prime \prime}$. The spectrophotometric standard Wolf 1346 was used to flux-calibrate the spectra.

We also observed these two fields on 2010 April 20 to obtain optical images with the Large Format Camera (LFC) on the Hale $5 \mathrm{~m}$ telescope at Palomar observatory. The two fields were easily covered by the mosaic camera with a field of view of 24 arcmin diameter. We used the $g^{\prime}$ and $i^{\prime}$ filters with a total exposure time of 1500 and $3000 \mathrm{~s}$, respectively. On readout, we binned $2 \times 2$, resulting in a pixel scale of 0.36 per pixel. The 
Table 1

Summary of WFC3 Imaging and Spectroscopy

\begin{tabular}{|c|c|c|c|c|c|c|}
\hline Field & $\begin{array}{l}\text { R.A. } \\
\text { (hms) }\end{array}$ & $\begin{array}{l}\text { Decl. } \\
\text { (dms) }\end{array}$ & $\begin{array}{c}\text { F110W } \\
\text { (s) }\end{array}$ & $\begin{array}{c}G_{102} \\
(\mathrm{~s})\end{array}$ & $\begin{array}{c}\text { F140W } \\
\text { (s) }\end{array}$ & $\begin{array}{c}G_{141} \\
(\mathrm{~s})\end{array}$ \\
\hline WISP1 & 010635.29 & +150853.8 & 884 & 4815 & 506 & 2609 \\
\hline WISP2 & 012510.02 & +213913.7 & 0 & 0 & 328 & 1906 \\
\hline WISP5* & 142706.64 & +575136.2 & 1034 & 5515 & 1034 & 5515 \\
\hline WISP6 & 015017.18 & +130412.8 & 609 & 3609 & 862 & 5015 \\
\hline WISP7* & 142705.91 & +575333.7 & 834 & 6318 & 1112 & 6224 \\
\hline WISP8 & 115151.62 & +544041.2 & 1662 & 9021 & 581 & 2509 \\
\hline WISP9 & 122944.31 & +074823.5 & 759 & 4612 & 684 & 3712 \\
\hline WISP10 & 092507.84 & +485703.0 & 631 & 3909 & 406 & 2209 \\
\hline WISP11 & 110217.38 & +105425.4 & 556 & 3709 & 456 & 2006 \\
\hline WISP12 & 120925.25 & +454319.8 & 1312 & 8221 & 606 & 3009 \\
\hline WISP13 & 010638.77 & +150826.2 & 556 & 3009 & 506 & 2409 \\
\hline WISP14 & 023456.80 & -040654.5 & 834 & 6215 & 481 & 2809 \\
\hline WISP15 & 140942.47 & +262156.0 & 1612 & 8321 & 531 & 2609 \\
\hline WISP16 & 023454.72 & -040642.5 & 1087 & 6921 & 584 & 2509 \\
\hline WISP18 & 122917.25 & +104400.6 & 534 & 3512 & 762 & 3824 \\
\hline WISP19 & 023454.29 & -040630.5 & 1187 & 8721 & 484 & 2809 \\
\hline WISP20 & 140941.15 & +262215.1 & 1815 & 8430 & 559 & 2812 \\
\hline WISP21 & 092755.77 & +602705.3 & 0 & 0 & 353 & 2006 \\
\hline WISP22 & 085244.99 & +030909.6 & 0 & 0 & 253 & 1806 \\
\hline WISP23 & 094316.12 & +052737.1 & 0 & 0 & 681 & 4115 \\
\hline WISP24 & 121841.90 & +295251.8 & 0 & 0 & 278 & 1806 \\
\hline WISP25 & 100842.49 & +071110.3 & 0 & 0 & 734 & 4115 \\
\hline WISP26 & 084517.91 & +225458.5 & 840 & 5521 & 384 & 2209 \\
\hline WISP27 & 113305.98 & +032802.2 & 1040 & 6218 & 384 & 2206 \\
\hline WISP28 & 093546.24 & +142748.5 & 0 & 0 & 634 & 3515 \\
\hline WISP29 & 120259.55 & +480521.2 & 0 & 0 & 328 & 1906 \\
\hline WISP30 & 102818.91 & +391714.1 & 0 & 0 & 253 & 1806 \\
\hline WISP31 & 084327.99 & +261639.7 & 0 & 0 & 659 & 4412 \\
\hline WISP32 & 130520.67 & -253805.5 & 0 & 0 & 484 & 3512 \\
\hline WISP33 & 100002.78 & +124456.4 & 959 & 6415 & 484 & 2809 \\
\hline WISP34 & 093458.06 & +020203.7 & 0 & 0 & 709 & 4215 \\
\hline WISP35 & 130346.90 & +295303.8 & 0 & 0 & 634 & 3812 \\
\hline WISP36 & 134031.52 & +412310.3 & 1237 & 8621 & 584 & 2809 \\
\hline WISP38 & 122513.33 & -024908.4 & 0 & 0 & 709 & 4312 \\
\hline WISP39 & 100938.23 & +300048.7 & 0 & 0 & 328 & 1706 \\
\hline WISP40 & 021620.12 & -390228.1 & 0 & 0 & 584 & 4112 \\
\hline WISP41 & 120828.31 & +453848.2 & 1084 & 6615 & 584 & 2909 \\
\hline WISP42 & 100104.36 & +502402.8 & 1812 & 10424 & 784 & 4012 \\
\hline WISP43 & 210407.62 & -072300.8 & 909 & 6315 & 434 & 2809 \\
\hline WISP44 & 111215.18 & +353655.5 & 0 & 0 & 734 & 4515 \\
\hline WISP45 & 123726.68 & +012518.2 & 0 & 0 & 796 & 4812 \\
\hline WISP46 & 223758.48 & -184205.4 & 0 & 0 & 328 & 1906 \\
\hline WISP46 & 223758.48 & -184205.4 & 0 & 0 & 328 & 1906 \\
\hline WISP47 & 131932.40 & +272640.0 & 0 & 0 & 587 & 3212 \\
\hline WISP49 & 144444.84 & +342745.9 & 1093 & 6018 & 406 & 2406 \\
\hline WISP50 & 222220.25 & +093636.8 & 0 & 0 & 328 & 1906 \\
\hline WISP51 & 151313.77 & +363322.0 & 0 & 0 & 278 & 2006 \\
\hline WISP52 & 133022.62 & +281101.0 & 0 & 0 & 634 & 4218 \\
\hline WISP53 & 151112.91 & +402539.3 & 0 & 0 & 253 & 1806 \\
\hline WISP54 & 154457.26 & +484523.2 & 0 & 0 & 303 & 2006 \\
\hline WISP56 & 161650.58 & +063643.6 & 0 & 0 & 684 & 4312 \\
\hline WISP57 & 123316.74 & +475327.5 & 0 & 0 & 659 & 4615 \\
\hline WISP59 & 155022.59 & +395919.2 & 0 & 0 & 734 & 4515 \\
\hline WISP61 & 230900.35 & -090717.7 & 0 & 0 & 709 & 4312 \\
\hline
\end{tabular}

Notes. Observation information for the fields analyzed in this paper. WISP5 and WISP7 have optical imaging in $g^{\prime}$ and $i^{\prime}$ bands with exposure times of 1500 and $3000 \mathrm{~s}$, respectively. The two fields were also covered by a slit mask with LRIS at Keck I for a total exposure time of 5400 s. 1". 2 wide slits were used.

typical seeing during observations was about $1^{\prime \prime}$. We adopted a five point dithering pattern with a $15^{\prime \prime}$ displacement. A summary of the observations is given in Table 1.
The images were corrected for bias and flat field using standard IRAF reduction packages. The flux calibration was performed by comparing the photometry of the field stars of our images with the those of the SDSS (York et al. 2000) that we obtained from the data release DR8. Finally, we aligned the ground-based frames to our IR ones using GEOMAP and GEOTRAN tasks in IRAF. In order to measure the object magnitudes in the optical images, we need to match the photometry aperture between ground-based and the HST images. We first convolve the WFC3 images with a Gaussian kernel to match the pointspread function (PSF) of the Palomar data. Then, we measure the flux in the convolved images using an aperture of five WFC3/IR pixels. The aperture size adopted is large enough to encompass most of the object flux for our compact sources, without being contaminated by nearby sources. We then compare the measured magnitudes to the AUTO magnitudes measured with SExtractor in the original WFC3 images. This gives us the aperture correction to apply to the magnitudes measured in the optical data.

\section{HIGH EQUIVALENT-WIDTH POPULATION}

The WISP Survey is particularly sensitive to high specific SFR (sSFR) and strong emission-line galaxies. In the fields we observed so far, we often see isolated emission lines in the grism frames, with either a faint continuum or no continuum at all. Such objects are easily identifiable by visually inspecting the final one-dimensional spectra. We only search part of the field, for which the zeroth-order information is available. To this aim, we have developed a flagging scheme in our reduction pipeline that marks the position of the zeroth order in the two-dimensional and one-dimensional spectra, to prevent their identification as emission lines. We then use a custom IDL procedure based on a least-square method to fit all the lines in each spectrum with a Gaussian and a polynomial continuum model.

After the line measurement, we have retained only galaxies with rest-frame EW higher than $200 \AA$. Examples of strong emission-line objects in our catalog are presented in Figure 1. The strong lines are typically $\mathrm{H} \alpha$ and [O III]. The sample reaches a $3 \sigma$ flux limit of $7 \times 10^{-17} \mathrm{erg} \mathrm{s}^{-1} \mathrm{~cm}^{-2}$ over a $180 \mathrm{arcmin}^{2}$ area, which is about three times the area surveyed in Atek et al. (2010). Both IR grisms are used to observe 24 fields $\left(80 \operatorname{arcmin}^{2}\right)$ over a wavelength range of $0.8-1.7 \mu \mathrm{m}$, and 30 fields $\left(100 \operatorname{arcmin}^{2}\right)$ are observed with $G_{141}$ only, covering a wavelength range 1.1-1.7 $\mu \mathrm{m}$. In Figure 2, we present the rest-frame EW distribution of the high-EW sample. It is well known that the $\mathrm{H} \alpha \mathrm{EW}$ is an indicator of the age of a galaxy (e.g., Leitherer et al. 1999). The $\mathrm{H} \alpha \mathrm{EW}$ indicates the ratio of the current SFR to the lifetime averaged SFR, also known as the birthrate parameter $b$ (Scalo 1986; Kennicutt 1998). The continuum luminosity is representative of the low-mass stars with a lifetime of several Gyr. The strength of the $\mathrm{H} \alpha$ line evolves on much shorter timescales, as it is the result of hot, short-lived $\mathrm{O}$ and $\mathrm{B}$ stars. A galaxy with a constant star formation will have a birthrate parameter of unity, while $b>1$ indicates that the current episode of star formation is stronger than the average SFR. Using Starburst99 models (Leitherer et al. 1999), we find that the $200 \AA$ limit on $\mathrm{EW}(\mathrm{H} \alpha)$ selects either young galaxies (less than $10 \mathrm{Myr}$ ) with instantaneous burst episode, or galaxies with continuous star formation older than a burst and less than $1 \mathrm{Gyr}$.

We note that for extremely high-EW lines, the continuum remains undetected in the grism spectra, making the EW 

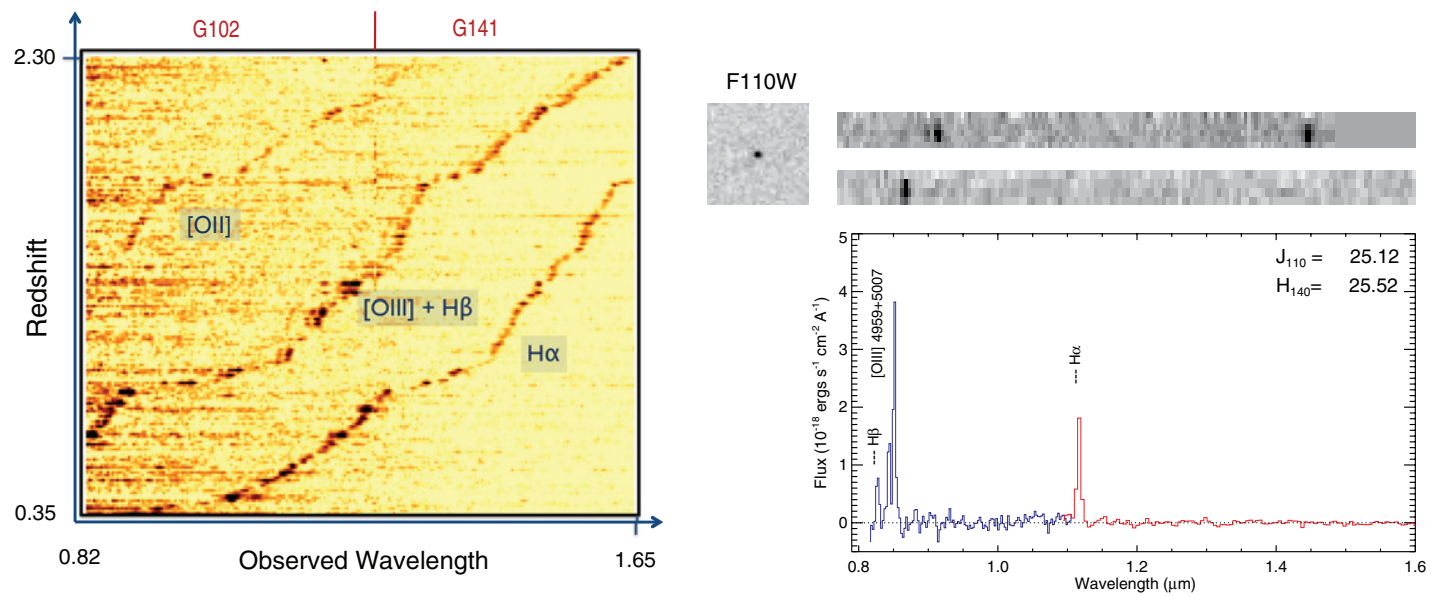

G102

G141

Figure 1. Strong emission-line galaxies across a wide range of redshift in the WISP Survey. Left: montage spectra of 176 high-EW emission-line galaxies stacked in the $Y$-axis direction with increasing redshift. Consequently, we see the emission lines we routinely detect in WISPS being shifted in the wavelength dimension. We note that the slope of the emission-line curve is less steep in some parts of this plot, i.e., we detect fewer objects per redshift bin, which is due the decreasing sensitivity toward the edge of the grisms. Right: an example of a galaxy at $z \sim 0.7$ showing strong emission lines. The direct F110W image and the $G_{102}$ and $G_{141}$ two-dimensional spectra are shown above the calibrated one-dimensional spectrum. The $J$ and $H$ magnitudes are also given in the inset. The object shows bright H $\alpha$ and [O III] lines at 0.85 and $1.12 \mu \mathrm{m}$, respectively, on top of a very faint continuum. The $\mathrm{H} \alpha$ line is detected in both grisms, in the overlapping region.

(A color version of this figure is available in the online journal.)

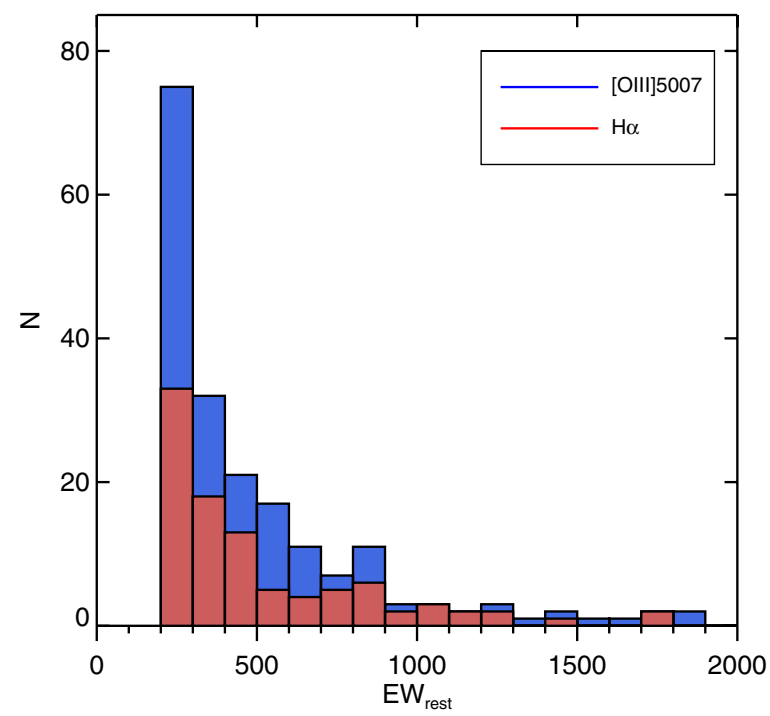

Figure 2. Rest-frame equivalent width distribution for objects with $E W \geqslant 200 \AA$ in the WISP Survey. The total number in each bin is divided into the [O III] $\lambda 5007$ line (presented in blue) and the $\mathrm{H} \alpha$ line (presented in red).

(A color version of this figure is available in the online journal.)

measurement highly uncertain. Given the low spectral resolution of the WFC3 grism, we are not able to separate the [N II] lines from $\mathrm{H} \alpha$. However, because the high-EW galaxies are likely metal deficient (see Section 7), we do not expect a large contribution from the [N II] lines. In fact, given an [O III] $\lambda 5007 / \mathrm{H} \beta$ ratio, one can infer the $\left[\mathrm{N}_{\mathrm{II}}\right] / \mathrm{H} \alpha$ ratio from the $[\mathrm{S}$ II $] / \mathrm{H} \alpha$ one. From Figure 1 of Kewley et al. (2006), and using the measured $\left[\mathrm{S}_{\mathrm{II}}\right] /([\mathrm{N} \mathrm{II}]+\mathrm{H} \alpha)$, we derive the $\left[\mathrm{S}_{\mathrm{II}}\right] / \mathrm{H} \alpha$ and then $\left[\mathrm{N}_{\mathrm{II}}\right] / \mathrm{H} \alpha$ for 17 high-EW galaxies. We find a median contribution of [N II] to $\mathrm{H} \alpha$ of about $8 \%$.

The strong [O III] $\lambda 5007$ line can also be the result of a photoionization dominated by harder radiation field from an active galactic nucleus (AGN). This will favor collisionally excited lines rather than recombination lines. In order to estimate the AGN contribution in the sample, we use the BPT (Baldwin, PXX, and TXX) diagnostic diagram proposed by Veilleux \&

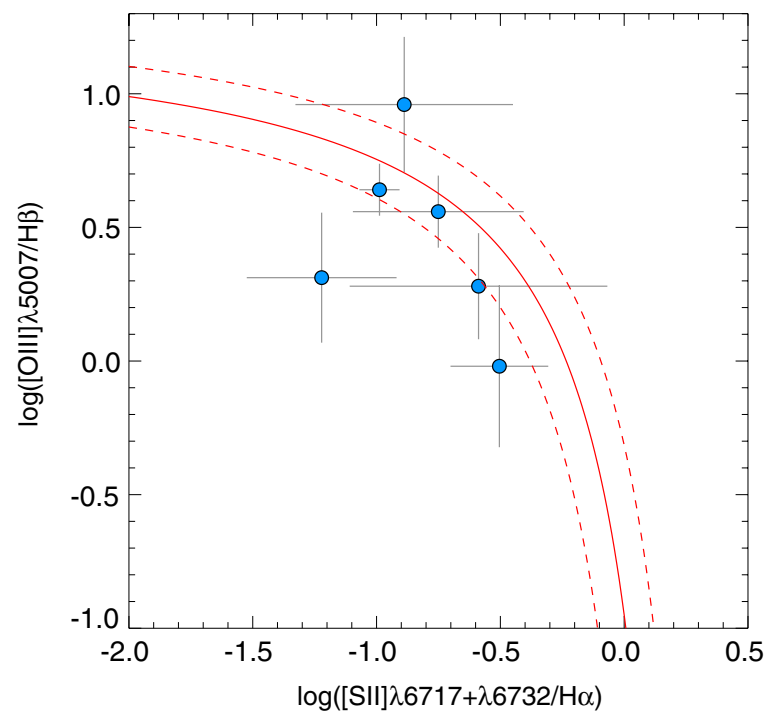

Figure 3. $[\mathrm{O}$ III $] \lambda 5007 \mathrm{H} \beta$ vs. [S II] $(\lambda 6717+6732) / \mathrm{H} \alpha$ diagnostic diagram. Six of our high-EW galaxies are represented with blue circles with $1 \sigma$ error bars. The solid red curve is the separation between star-forming galaxies and AGNs of Kewley et al. (2001). The red dashed lines show typical model uncertainties of \pm 0.1 dex.

(A color version of this figure is available in the online journal.)

Osterbrock (1987) using [O III] $\lambda 5007 / \mathrm{H} \beta$ versus [S II] $\lambda(6717$ $+6732) / \mathrm{H} \alpha$ line ratios, where $\mathrm{H} \alpha$ is corrected for [N $\mathrm{NII}]$ contamination following the procedure described above. We note that using the BPT diagram to classify low-metallicity dwarf galaxies with high sSFR can be somewhat ambiguous (Brinchmann et al. 2008; Izotov \& Thuan 2008). Brinchmann et al. (2008) show that high-sSFR galaxies may have higher values of the ionization parameter than normal star-forming galaxies, and tend to be offset in the BPT diagram toward the AGN region.

The classification of the six objects is presented in Figure 3 . We identified one AGN in a subsample of six galaxies that fall in the right redshift range $(0.7<z<1.4)$ and have detectable $\mathrm{H} \beta$ and [S II] lines. This represents a fraction of about $17 \%$, although 
the statistics are too small to derive a secure AGN fraction for the entire sample. This is consistent with the fraction found in $z \sim 0.3-2$ Ly $\alpha$ emitters (Atek et al. 2009; Scarlata et al. 2009; Nilsson et al. 2009; Cowie et al. 2010, 2011), and what is locally found in emission-line galaxy samples (e.g., Hao et al. 2005).

This high-EW selection is comparable to that of groundbased narrowband surveys (Kakazu et al. 2007). Also, using broadband excess, Shim et al. (2011) identified strong $\mathrm{H} \alpha$ emitters in the redshift range of $3.8<z<5.0$ that have mostly $\mathrm{EW}(\mathrm{H} \alpha)>200 \AA$. A total of 176 objects satisfy the high-EW criterion, spanning a redshift range of $0.35<z<2.3$, which results in a surface density of one object per square arcmin. We are also interested in the redshift evolution of the space density of the high-EW galaxies. In order to compare our results with the local universe, we analyzed the SDSS sample of emissionline galaxies at $z<0.1$. We used the spectral measurements in catalog of the MPA-JHU DR7 release ${ }^{10}$ to derive absolute $R$ magnitudes from the SDSS apparent $r$ magnitudes using Jester et al. (2005) conversion solutions. A passive evolution correction was applied to the WISP sample in order to compare the space densities at a fixed stellar mass (e.g., Sargent et al. 2007). We applied an absolute magnitude cut at $-21<M_{R}<-18.5$ in the redshift interval $0.05<z<0.1$. The same absolute magnitude cut was applied to the high-EW sample. In the end, the space density of galaxies with EW $>200 \AA$ is about $5.5 \times 10^{-6} \mathrm{Mpc}^{-3}$ in the SDSS, and about $7 \times 10^{-5} \mathrm{Mpc}^{-3}$ in WISP. This represents an evolution of more than a factor of 10 in the number density of strong emission-line galaxies between $z \sim 1.3$ and $z \sim 0$.

\section{CONTRIBUTION OF EMISSION LINES TO BROADBAND PHOTOMETRY}

To quantify the contribution of nebular lines to the total broadband flux density, we have calculated synthetic flux densities from our spectra using the transmission curves of $J_{110}$ and $H_{140}$ filters and the continuum-subtracted emission lines only, which were then compared to the total flux density. The result is shown in Figure 4 where we plot the contribution of the line flux density to the $J_{110}$ and $H_{140}$ total flux densities as a function of the magnitudes in these filters. The nebular contribution can represent most of the broadband flux density of the galaxy, i.e., more than $50 \%$, which translates into more than $0.75 \mathrm{mag}$. The distribution has a median value of about $25 \%$, which represents 0.3 mag contribution from the emission lines. The presence of such strong emission lines in the restframe optical spectra can have several implications on the study of high-redshift galaxy populations.

\subsection{Contamination of High-redshift Galaxy Samples}

In order to select very high redshift galaxies, the Lyman break technique exploits the continuum drop blueward of Ly $\alpha$ caused by the intervening intergalactic medium (IGM) absorption. By using a set of broadband filters, one can sample the continuum of the galaxy to search for a detection in the red band and an absence of signal in the bluer bands. This method has been extensively used to select $z \sim 7-10$ candidates using, in particular, the WFC3 IR camera onboard HST (Oesch et al. 2010; Bunker et al. 2010; Yan et al. 2010; Bouwens et al. 2011a; Lorenzoni et al. 2011). Other groups have identified high- $z$ sources in the WFC3 imaging data set by using the SED fitting

\footnotetext{
$\overline{10}$ http://www.mpa-garching.mpg.de/SDSS/DR7/
}

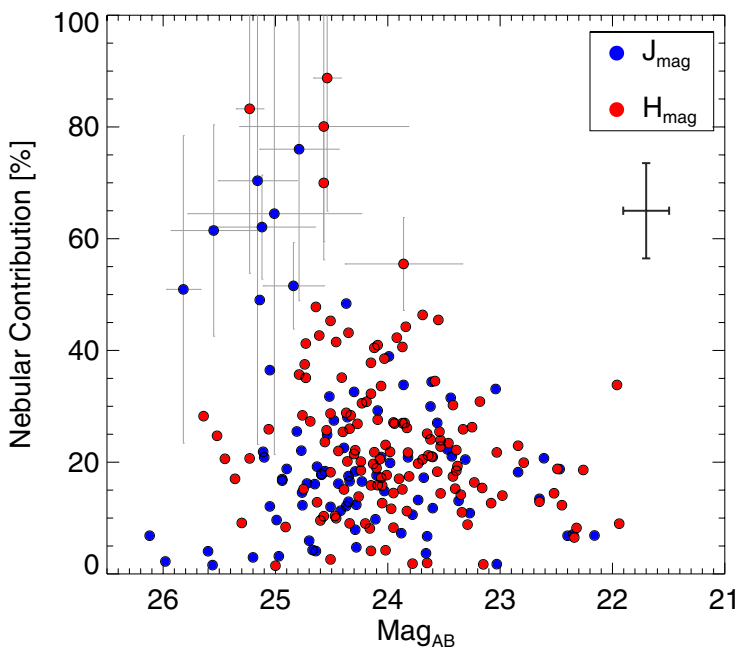

Figure 4. Contribution of the nebular line flux density to the total broadband flux density in the $J$ and $H$ bands as a function of the total magnitude for objects with $\mathrm{EW}_{\text {rest }}>200 \AA$. This quantity is calculated by comparing the synthetic flux density of the emission lines only to the total flux density in the filter. The contribution to the F110W filter flux density is presented in blue and the F140W in red. We plot error bars for objects that have a nebular contribution higher than $50 \%$. For object with less than $50 \%$ contribution, we plot instead averaged error bars on the top right of the figure.

(A color version of this figure is available in the online journal.)

approach to obtain photometric redshift distributions (McLure et al. 2010; Finkelstein et al. 2010). Also, in order to probe the bright end of the luminosity function (LF) at $z \sim 7$, a wide area coverage is necessary. Two HST observing programs started recently to search for bright $z>7$ dropout candidates using pure parallel WFC3 NIR imaging of random fields: BoRGs (Brightest of Reionizing Galaxies; Trenti et al. 2011) and HIPPIES (Hubble Infrared Pure Parallel Imaging Extragalactic Survey; Yan et al. 2011).

However, low-redshift sources can have similar colors to those of high- $z$ galaxies and therefore satisfy the dropout selection criteria. The possible contamination from cool dwarfs, transient objects, old galaxies, or spurious detections has been addressed in previous studies (e.g., Bouwens et al. 2011a). We consider in this work another potential source of contamination: the strong emission-line galaxies at lower redshift. Since the WISP emission-line galaxy sample is spectroscopically selected over a wavelength range of $0.8-1.7 \mu \mathrm{m}$, we were naturally interested in the case of $z \gtrsim 7$ galaxies, so called $Y$ and $J$ dropouts. We note that the wide shallow observations of Trenti et al. (2011) and Yan et al. (2011) use the $Y_{098}$ filter, whereas the deep programs use $Y_{105}$. This can slightly change the results presented hereafter because of the different width of the filters and the wavelength position of the emission lines.

In order to estimate the contamination of such sources to the dropout selection, we use the same filter set used by the wide surveys, i.e., $Y_{098}, J_{125}$, and $H_{160}$, and compare their colors. The dominating contributions that affect the broadband colors are [O III] $\lambda \lambda 5007,4959$ and $\mathrm{H} \alpha$ emission lines (cf. left panel of Figure 5). In the right panel of Figure 5, we examine the location of the emission lines and their relative contribution to the total flux density of the filters as a function of redshift. Depending upon the wavelength position of the emission lines, a galaxy can appear to have a wide range of IR colors and scatter into the high- $z$ dropout selection window.

Figure 6 shows the $Y_{098}-J_{125}$ versus $J_{125}-H_{160}$ color-color diagram commonly used to isolate high $z$ candidates populated 

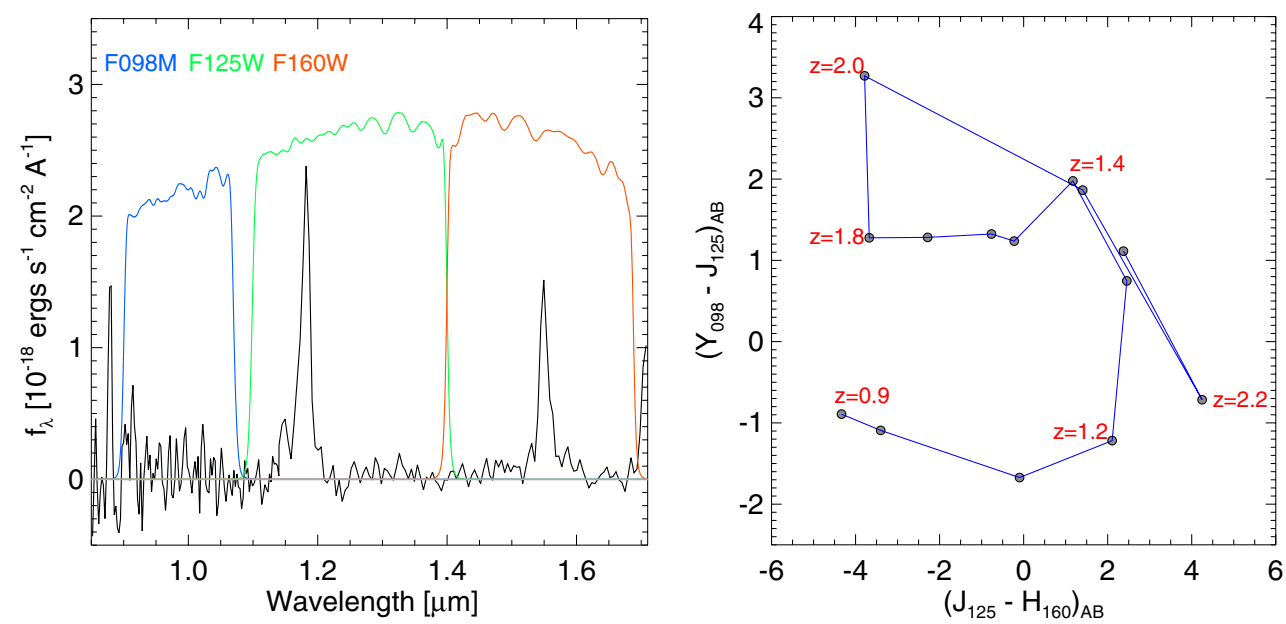

Figure 5. Contamination of the selection of high-redshift dropout galaxies by lower-redshift sources with strong emission lines. Left: example of a high-EW emissionline object, WISP42_115 at redshift $z=1.36$. The throughput curves of the 098M, F125W, and F160W filters used for the synthetic flux calculations are over-plotted. Right: $Y_{098}-J_{125}$ vs. $J_{125}-H_{160}$ color track of strong emission-line galaxy as a function of redshift. An example of a high-EW galaxy spectrum is used to calculate synthetic magnitudes in the IR bands, with $\mathrm{EW}([\mathrm{O}$ II $] \lambda 3727,[\mathrm{O}$ III] $\lambda 5007, \mathrm{H} \alpha) \sim(200,800,550)$. Beyond the wavelength limits of the WISP spectrum, a flat continuum in $f_{v}$ is assumed.

(A color version of this figure is available in the online journal.)

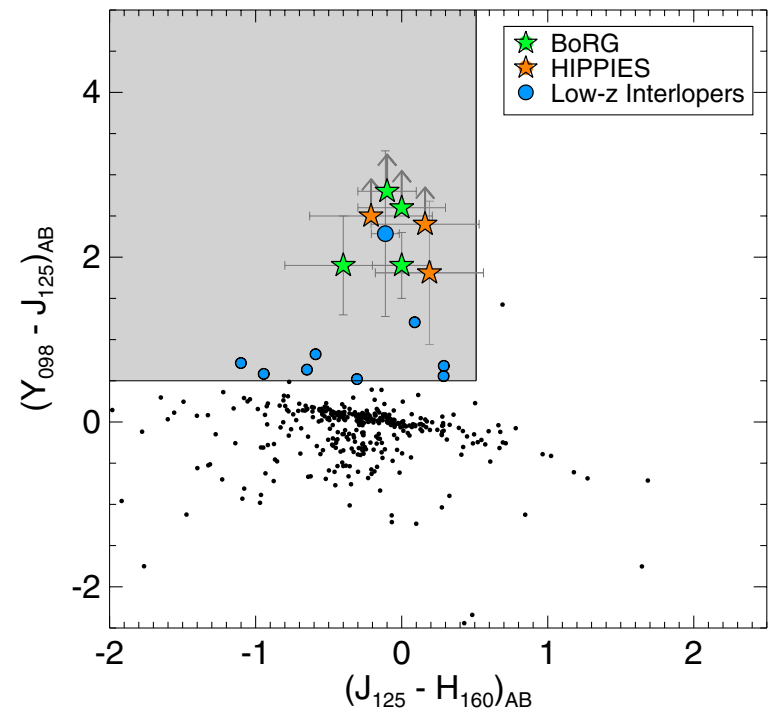

Figure 6. $Y_{098}-J_{125}$ vs. $J_{125}-H_{160}$ color-color diagram for the high-EW sample of galaxies. The magnitude in each band is derived from synthetic flux calculation using the grism spectra and the WFC3 filter curves. The shaded region denotes the selection window of $z \sim 8 Y_{098}$-dropout surveys, which satisfy $Y_{098}-J_{125}>0.5$ and $J_{125}-H_{160}<0.5$. The galaxies marked in blue circles satisfy this general color-color-selection criterion, whereas the rest of the sample is shown with black points. In addition, the galaxy represented with a bigger blue circle satisfies $Y_{098}-J_{125}>1.3$ and $J_{125}-H_{160}<0.3$ used by the wide surveys. We compare this galaxy with the $Y_{098}$-dropout candidates of Yan et al. (2011) and Trenti et al. (2011), shown by orange and green stars, respectively, and whose color criteria are detailed in the text. The error bars indicate $1 \sigma$ uncertainties, while the arrows represent lower limits.

(A color version of this figure is available in the online journal.)

with the high-EW galaxies. We find a large dispersion in both $J_{125}-H_{160}$ and $Y_{098}-J_{125}$ colors, and we observe galaxies that fall in the high- $z$ selection window (gray region) that denotes the color selection $J_{125}-H_{160}<0.5$ and $Y_{098}-J_{125}>0.5$ (e.g., Bouwens et al. 2011b).

The contamination of $z \sim 8$ galaxy candidates by low- $z$ interlopers with strong emission lines has been investigated theoretically by Taniguchi et al. (2010). These authors used SED models to predict the strength of the emission lines from the ionizing continuum, and then examined whether their synthetic spectra satisfy the selection criterion of $z \sim 8$ galaxies. The [O III] $\lambda 5007 \mathrm{EW}$ in their models varies from $\sim 10^{3} \AA$ to $\sim 100 \AA$ for ages of 1-100 Myr. They conclude that the contribution of the emission lines is too small to meet the color requirement. We measure EW > $1000 \AA$ in $z \sim 1-2$ galaxies, however, and these do satisfy the $Y$-dropout selection as we can see in Figure 6. However, one of the main conditions used to rule out low- $z$ interlopers in such surveys is the non-detection in the optical domain. To this end, HUDF observations (Bouwens et al. 2011b; Bunker et al. 2010; Yan et al. 2010) make use of Advanced Camera for Surveys (ACS) data in several optical bands which are at least 1 mag deeper than IR images. This makes the contamination from low- $z$ strong line emitters very unlikely, unless the extinction is very high. We were able to measure the reddening from the $\mathrm{H} \alpha / \mathrm{H} \beta$ ratio for one of the interlopers of Figure 6 (purple circles) and found a small value of $E(B-V) \sim 0.05\left(A_{v} \sim 0.15\right)$. Also, in the rest of the high-EW sample, objects for which $\mathrm{H} \alpha$ and $\mathrm{H} \beta$ are available show little extinction. It is also possible that this is the result of a selection effect in the sense that we are missing the dusty galaxies where the $\mathrm{H} \beta$ line remains undetectable because it would be fainter than our flux limit.

On the other hand, the wide pure parallel surveys BoRG and HIPPIES do not use such deep optical data to discard these interlopers. Indeed, the UVIS observations in the F606W or F600LP bands reach a depth comparable to the IR. The colorselection criteria used in these studies are $Y_{098}-J_{125}>1.75$ and $J_{125}-H_{160}<0.02+0.15 \times\left(Y_{098}-J_{125}-1.75\right)$ for Trenti et al. (2011), and $Y_{098}-J_{125}>1.35$ and $J_{125}-H_{160}<0.3$ for Yan et al. (2011), respectively. We plot in Figure 6 the colors of the high-EW galaxies (in blue) together with the samples of $z \sim 8$ candidates selected in the two parallel surveys (orange and green). When applying the respective color criteria of these two surveys, and given the uncertainties, we end up with one interloper in both the BoRG and HIPPIES samples. In this section, we used 24 fields that have both $G_{102}$ and $G_{141}$ observations, which yields an effective survey area of about 
Table 2

Broadband Photometry of the Sample

\begin{tabular}{|c|c|c|c|c|c|c|c|}
\hline Object & $z$ & $\begin{array}{c}g^{\prime} \\
(\mathrm{mag})\end{array}$ & $\begin{array}{c}i^{\prime} \\
(\mathrm{mag})\end{array}$ & $\begin{array}{c}J \\
(\mathrm{mag})\end{array}$ & $\begin{array}{c}J_{\text {corr }} \\
(\mathrm{mag})\end{array}$ & $\begin{array}{c}H \\
(\mathrm{mag})\end{array}$ & $\begin{array}{c}H_{\text {corr }} \\
(\mathrm{mag})\end{array}$ \\
\hline WISP5_53 & 1.94 & $22.98(0.02)$ & $22.52(0.04)$ & $22.43(0.14)$ & 22.46 & $22.14(0.01)$ & 22.30 \\
\hline WISP5_73 & 0.88 & $24.30(0.06)$ & $23.28(0.05)$ & $23.04(0.19)$ & 23.58 & $23.03(0.01)$ & 23.37 \\
\hline WISP5_108 & 1.36 & $24.76(0.08)$ & $24.11(0.10)$ & $23.62(0.25)$ & 23.85 & $23.57(0.02)$ & 24.03 \\
\hline WISP5_170 & 1.62 & $25.36(0.14)$ & $25.19(0.28)$ & $24.54(0.38)$ & 24.90 & $24.61(0.04)$ & 25.33 \\
\hline WISP5_217 & 1.93 & $24.93(0.09)$ & $24.55(0.16)$ & $24.97(0.45)$ & 25.04 & $24.12(0.02)$ & 24.74 \\
\hline WISP5_230 & 0.70 & $25.93(0.23)$ & $25.84^{\mathrm{a}}(0.16)$ & $25.12(0.48)$ & 26.17 & $25.52(0.08)$ & 25.53 \\
\hline WISP7_140 & 1.20 & $25.00(0.10)$ & $24.70(0.18)$ & $23.99(0.29)$ & 24.52 & $24.05(0.04)$ & 24.24 \\
\hline WISP7_145 & 2.04 & $24.65(0.07)$ & $24.24(0.12)$ & $24.04(0.30)$ & 24.24 & $23.67(0.02)$ & 23.85 \\
\hline WISP7_152 & 1.22 & $25.03(0.10)$ & $24.43(0.14)$ & $24.09(0.30)$ & 24.46 & $24.07(0.03)$ & 24.32 \\
\hline
\end{tabular}

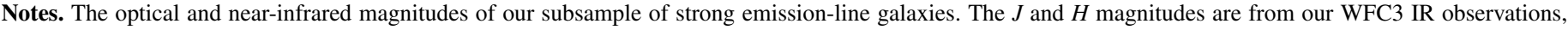

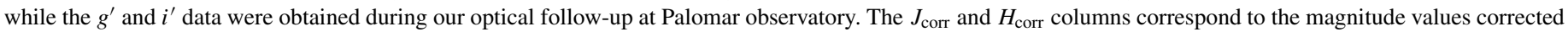

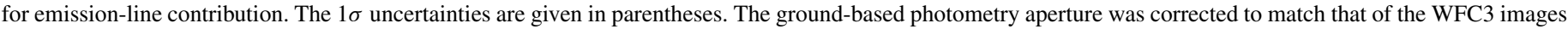
(cf. Section 3). Magnitudes are in the AB system.

${ }^{\text {a The }} i^{\prime}$-band flux of this object was corrected (by $1.3 \mathrm{mag}$ ) for the contamination of strong emission lines using the LRIS spectrum.

$80 \operatorname{arcmin}^{2}$. When we scale this area to the above surveys, we find that the contamination from such sources represents about one object in every 17 fields observed by the wide shallow surveys. This is assuming the same number density of such sources at the depth of the wide surveys, because their photometric observations go deeper than our spectroscopic survey. This result is also consistent with the prediction of Trenti et al. (2011) that estimated a $30 \%$ contamination rate in their survey.

To show the necessity of deep optical imaging, we have estimated the expected flux in the optical bands of these interlopers using the best-fit SEDs of the high-EW galaxies presented in Section 5.2. Using the filter throughputs, we calculated the magnitudes in the F606W and F600LP optical bands. We find that almost all the galaxies have red $V_{606}-Y_{098}$ colors ranging from 0.1 to $0.9 \mathrm{mag}$. The same red colors are obtained when we use the F600LP filter. These results are also confirmed by the $g^{\prime}-J$ and $i^{\prime}-J$ colors observed for these objects (see Table 2). While some of the interlopers that satisfy the IR color criteria can be identified in the UVIS filters, a mean value of $V_{606}-Y_{098} \sim 0.6$ in our results suggests that $\sim 1 \mathrm{mag}$ deeper imaging is needed in the optical in order to rule out the contamination from all the low- $z$ high-EW galaxies. In this sense, the ongoing wide survey of the Cosmic Assembly NearIR Deep Extragalactic Legacy Survey (CANDELS; Grogin et al. 2011) will have appropriately deep optical imaging in multiple HST/ACS bands.

The high- $z$ narrowband surveys of Ly $\alpha$ emitters (LAEs) can also be, to some extent, subject to the contamination of these strong low $z$ emitters. The selection method relies on the flux excess detected in the narrow band relative to the broadband flux, and selects candidates that have rest-frame EW higher than $\sim 40 \AA$. Obviously, the galaxies presented in this work have much larger EW values, and the narrowband excess can be attributed in some cases to these lower redshift interlopers. However, at redshift $z \sim 6.5$ for instance, Ouchi et al. (2010) and Kashikawa et al. (2011) use also the color blueward of the Ly $\alpha$ emission to detect the Lyman break of the NB921-selected galaxies. This additional criterion significantly reduces the low$z$ contamination fraction. Using $R-i>1$ and $i-z>1.2$, Ouchi et al. $(2008,2010)$ found in their spectroscopic follow-up that such contamination is very small for their sample of $z \sim 5.7$ and $z \sim 6.6$ candidates, respectively. Using similar constraints, Kashikawa et al. (2011) report $81 \%$ (70\%) confirmation rate for their $z=6.5$ (5.7) LAE candidates. Combining the color check blueward of Ly $\alpha$ with the narrowband selection should be sufficient to identify the low- $z$ high-EW sources.

\subsection{Effects on Age and Mass Estimate of High-z Galaxies}

While most stellar population synthesis models do not include the nebular emission lines, some effort has been devoted recently to take into account the impact of such contribution on the SED analysis of high- $z$ galaxies. Eyles et al. (2007) used the UV SFR to assess the contribution of optical emission lines to their Spitzer IRAC photometry and their effect on the SED fitting of $z \sim 6$ galaxies. More recently, Finkelstein et al. (2011) corrected for the optical emission lines in their fitting of two LAEs at $z \sim 2.3$ (see also McLinden et al. 2011). Schaerer \& de Barros (2009) treated the effect of nebular emission (lines and continua) in a self-consistent way by predicting the absolute line intensities from the ionizing continuum of the template SED. They showed that neglecting the nebular component could lead to overestimating the age of $z \sim 6$ galaxies by a factor of 3 . This theoretical prescription has been followed in several papers (e.g., Ono et al. 2010; Watson et al. 2010; Raiter et al. 2010; Finlator et al. 2011; McLure et al. 2011; Inoue 2011) dealing with SED modeling.

Using the sample of very high EW objects, we empirically study the impact of nebular emission lines on the SED fitting of galaxies in general, and on the age and mass estimates in particular. We selected nine galaxies from fields WISP5 and WISP7 that have optical follow-up in $g$ and $i$ bands obtained with the $5 \mathrm{~m}$ Hale telescope at Palomar observatory, in addition to the $J$ and $H$ photometry from the WISPS IR observations.

To model the stellar population properties of these galaxies, we use the FAST code (Kriek et al. 2009) to fit Bruzual \& Charlot (2003) synthesis models to the continuum magnitudes. We assume exponentially declining SFH, a metallicity of $Z=0.02$, and a Chabrier (2003) initial mass function (IMF). The fitting results are presented in Figures 7, 8, and 12. The black curve is the model spectrum fit to the raw observed magnitudes. The red curve is the model fit to the magnitudes corrected for the contamination of nebular emission lines following the procedure detailed in Section 5.1. In addition, we used the LRIS spectrum to correct the $i^{\prime}$-band magnitude of WISP5_230. Depending on the wavelength location and the strength of the contributing emission lines, the difference between the models varies dramatically. 

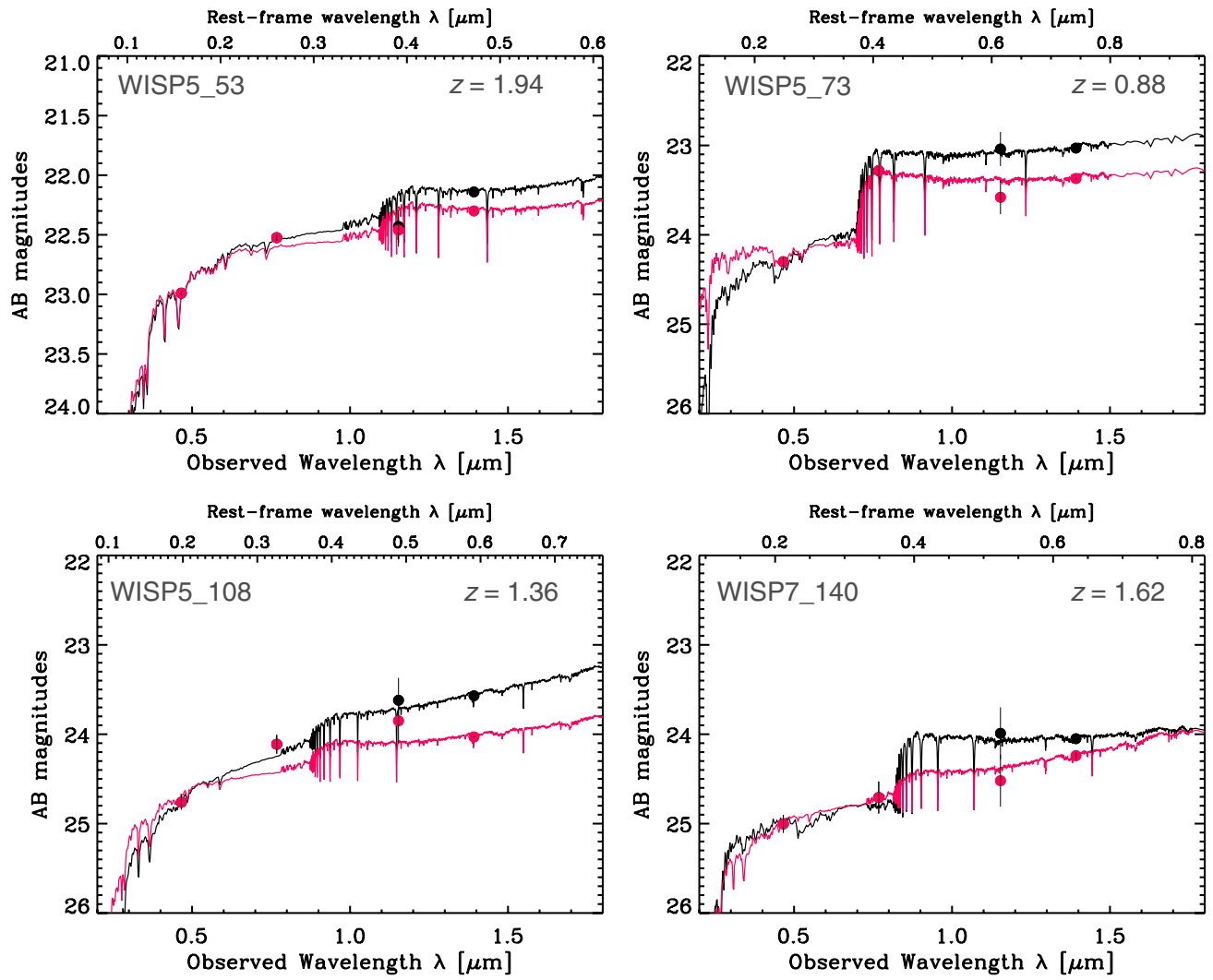

Figure 7. SED fitting of the high-EW galaxies. The black points represent the observed magnitudes in the $g^{\prime}, i^{\prime}, J$, and $H$ bands, while the red points are the magnitudes corrected for the contamination of nebular lines following the procedure explained in the text. The error bars show $1 \sigma$ uncertainties. The black and red curves are the best-fit models to the raw and corrected magnitudes, respectively. We used Bruzual \& Charlot (2003) models assuming exponentially declining star formation history, $0.02 Z_{\odot}$ metallicity, and Chabrier (2003) IMF.

(A color version of this figure is available in the online journal.)
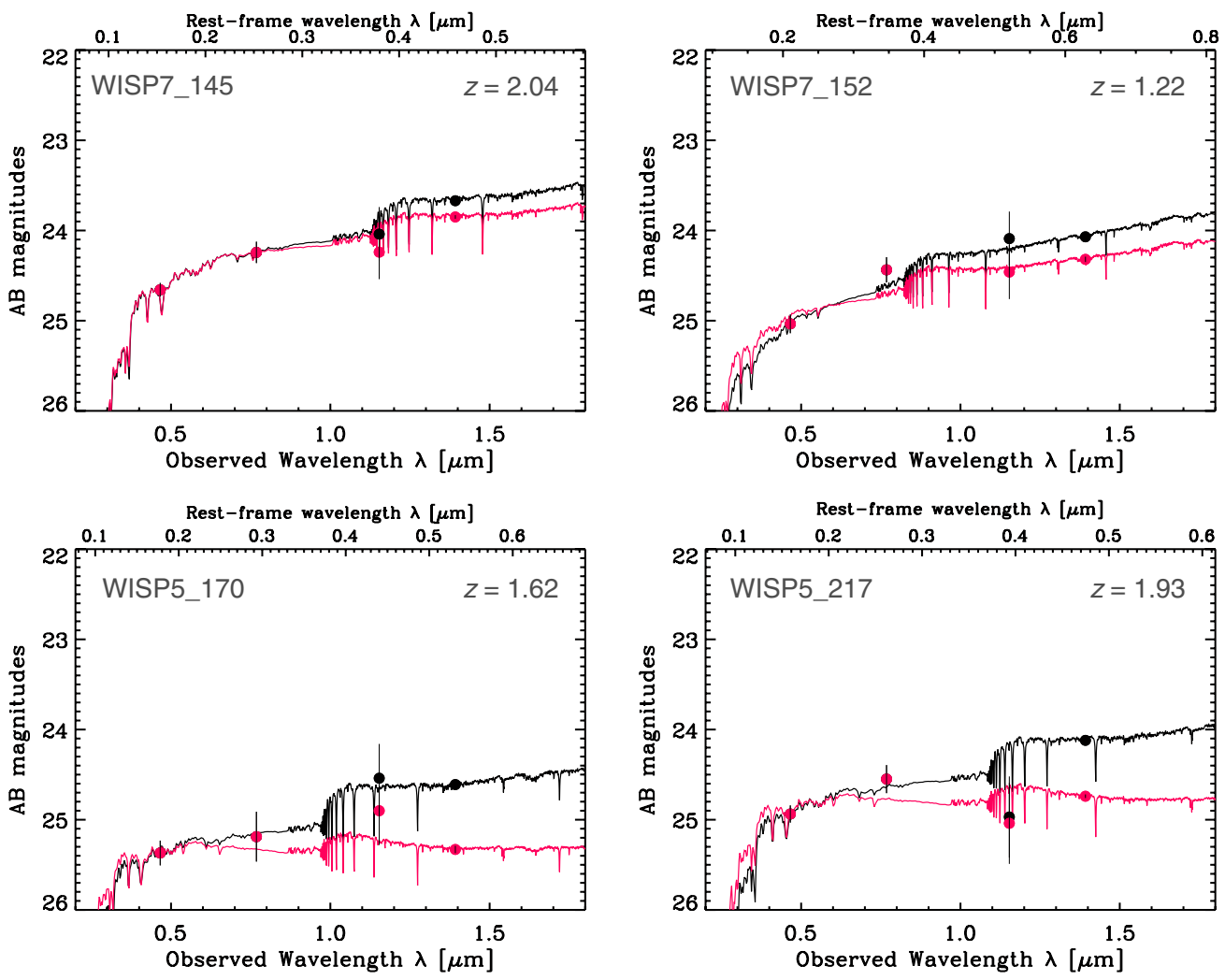

Figure 8. Same as Figure 7.

(A color version of this figure is available in the online journal.) 
Table 3

Impact of Emission Lines on the Physical Properties

\begin{tabular}{|c|c|c|c|c|c|c|}
\hline Object & $A_{v}$ & $\begin{array}{c}\log (\mathrm{SFR}) \\
\left(M_{\odot} \mathrm{yr}^{-1}\right)\end{array}$ & $\begin{array}{c}\log (\text { Age }) \\
(y r)\end{array}$ & $\begin{array}{c}\log (\text { Age (Corr) }) \\
(\mathrm{yr})\end{array}$ & $\begin{array}{c}\log \text { (Mass) } \\
\left(M_{\odot}\right)\end{array}$ & $\begin{array}{c}\text { Mass (Corr) } \\
\left(M_{\odot}\right)\end{array}$ \\
\hline WISP5_53 & 1.20 & 2.58 & 7.00 & 7.00 & 9.64 & 9.52 \\
\hline WISP5_73 & 0.20 & -0.90 & 8.20 & 8.40 & 9.08 & 8.91 \\
\hline WISP5_108 & 1.60 & 1.83 & 7.00 & 7.00 & 8.89 & 8.56 \\
\hline WISP5_170 & 0.70 & 0.78 & 8.20 & 7.00 & 8.90 & 8.00 \\
\hline WISP5_217 & 0.80 & 1.23 & 8.00 & 7.00 & 9.21 & 8.35 \\
\hline WISP5_230 & 0.00 & -1.00 & 8.30 & 8.20 & 7.81 & 7.67 \\
\hline WISP7_140 & 0.20 & -0.54 & 8.10 & 7.20 & 8.89 & 8.43 \\
\hline WISP7_145 & 1.00 & 1.63 & 7.90 & 7.30 & 9.45 & 9.09 \\
\hline WISP7_152 & 1.50 & 1.57 & 7.00 & 7.00 & 8.55 & 8.34 \\
\hline
\end{tabular}

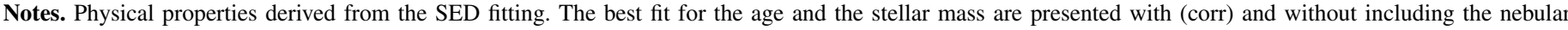
emission lines.

In Table 3, we compare the stellar population properties derived from the two model fits. It is shown that not accounting for the contribution of emission lines can significantly affect the age and the stellar mass estimates in these galaxies. Specifically, the age and the mass of individual objects can be overestimated by an order of magnitude. We show for instance that, based on the observed magnitudes, the galaxy WISP5_170 has an age of $t \sim 160 \mathrm{Myr}$ and a mass of $M^{\star} \sim 8 \times 10^{8} M_{\odot}$. Once corrected for the nebular emission, the age and mass are revised to $t \sim 10 \mathrm{Myr}$ and $M^{\star} \sim 10^{8} M_{\odot}$, respectively. Considering all the objects with varying emission-line intensities, we derive a mean age for the sample of $t_{\text {mean }} \sim 100 \mathrm{Myr}$ and a mass of $M_{\text {mean }}^{\star} \sim 1.4 \times 10^{9} M_{\odot}$. Correcting for emission lines brings these values down to $t_{\text {mean }} \sim 55 \mathrm{Myr}$ and a mass of $M_{\text {mean }}^{\star} \sim 7.3 \times 10^{8} M_{\odot}$. This change represents an average correction factor of 2. For comparison, Finkelstein et al. (2011) derived for their two objects at $z \sim 2.5$ a correction factor of $(2,6)$ for the mass and a factor of $(10,1)$ for the age. We note that the extinction $A_{v}$ can also be slightly overestimated if no correction is applied.

It is clear that not accounting for nebular emission lines when modeling their SEDs can introduce large errors in the derived age and stellar masses of star-forming galaxies at all redshifts. Deriving the physical properties of higher-redshift galaxies is much more problematic. Galaxies at $z>6$ will be younger, more metal deficient, and will exhibit higher EWs. Moreover, the contribution of emission lines to the broadband filters increases as $(1+z)$. However, this effect is mitigated by the fact that commonly used broad photometric bands increase in width when going redder. This will dilute the line in this large wavelength band and decrease the EW contribution. This empirical demonstration shows the need for careful SED modeling by systematically accounting for nebular emission lines.

\section{HIGH SPECIFIC STAR FORMATION RATE GALAXIES}

The correlation between the SFR of galaxies and their stellar mass $M^{\star}$ or their luminosity and metallicity has been studied extensively, especially at low redshift. Observationally, it has been investigated at different redshifts (Brinchmann et al. 2004; Erb et al. 2006a; Noeske et al. 2007b; Elbaz et al. 2007; Daddi et al. 2007; Damen et al. 2009; Labbé et al. 2010). This SFR $-M^{\star}$ relationship is sometimes called the galaxy main sequence (Noeske et al. 2007b) as a reference to the stellar main sequence. While an SFR- $M^{\star}$ relation is exhibited at all redshifts, it evolves in the sense that galaxies of a given stellar mass have higher SFRs at higher redshifts. The evolution of the SFR as a function of $M^{\star}$ is of particular interest as a test of galaxy evolution models. The SFR $-M^{\star}$ correlation is indeed predicted by cosmological hydrodynamic simulations (Finlator et al. 2011), and Bouché et al. (2010) argue that smooth gas accretion is responsible for this trend (see also Davé et al. 2011)

However, at high redshift, the SFR $-M^{\star}$ correlation has been limited to a relatively high mass range. The techniques used for the selection of galaxies include mostly the optical or NIR colors and UV continuum (e.g., Steidel et al. 1996, 2004; Daddi et al. 2004; Papovich et al. 2006; Shapley et al. 2005; Erb et al. 2006a; Schiminovich et al. 2007). Unfortunately, this misses a population of star-forming galaxies with faint continua. Photometric redshifts have also been used to identify high- $z$ galaxies, but remain unreliable at faint magnitudes because of photometric uncertainties and emission-line contribution. While the most massive galaxies are offset to lower sSFRs (the SFR per unit stellar mass) in the SFR $-M^{\star}$ plane, our sample selection targets precisely galaxies with higher sSFRs that would lie in the upper left corner of this plane.

In Figure 9, we present the SFR measured from emission lines as a function of the stellar mass for nine of the highEW sources for which we obtained optical follow-up imaging. The SFR is derived from the $\mathrm{H} \alpha$ flux using Kennicutt (1998) calibration. No dust correction was applied, as we were able to measure $\mathrm{H} \alpha$ and $\mathrm{H} \beta$ lines in five of these galaxies that were consistent with no, or little, extinction. We corrected the $\mathrm{H} \alpha$ flux for [ $\mathrm{N}$ II] contamination using the procedure described in Section 4. For high-redshift galaxies selected by their strong [O III] $\lambda 5007$ line, and where the $\mathrm{H} \alpha$ line is outside the $G_{141}$ window, we also have a detectable [O II] $\lambda 3727$ line. Likewise, we apply the Kennicutt (1998) prescription to convert the [O II] flux into SFR. The stellar masses were derived from SED fitting of the optical and NIR observations of the galaxies using Bruzual \& Charlot (2003) spectral synthesis models with exponentially declining star formation $e^{-t / \tau}$ with $t$ and $\tau$ as free parameters, a Chabrier (2003) IMF, and metallicity of $Z=0.02$.

It can be seen from Figure 9 that these galaxies have a higher sSFR than the sequence of normal star-forming galaxies at the same redshift derived in previous studies. They probe a mass range that has never been explored before. We divided the highEW sample into two bins of redshifts below and above $z=1.3$ drawn in blue and red, respectively. While Daddi et al. (2007) derive a tight SFR $-M^{\star}$ correlation for $z \sim 2$ galaxies in Great Observatories Origins Deep Survey (GOODS), with a dispersion 


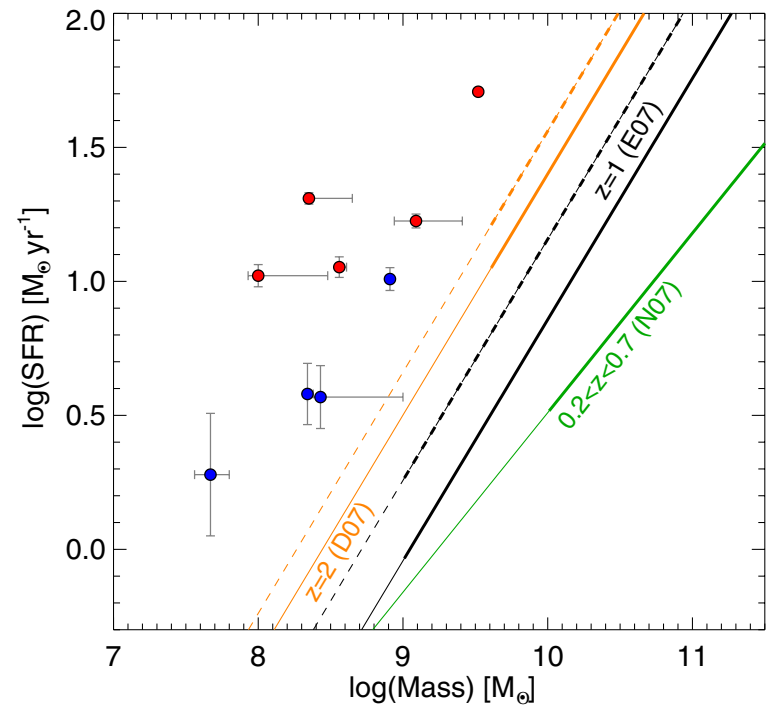

Figure 9. The star formation rate as a function of the stellar mass. The SFR is derived from the optical emission lines and the stellar masses from the SED fitting of the optical and NIR photometry (cf. the text for details). Objects located at $z>1.3$ are shown with red circles and objects at $z<1.3$ with blue circles. The $1 \sigma$ uncertainties on the mass and SFR are also overplotted. The lines denote previous results of several studies of the SFR-mass correlation: the orange line is the fit to $z=2$ sample of Daddi et al. (2007) with the dashed line representing the typical dispersion of 0.16 dex. The black line is for galaxies at $z=1$ from Elbaz et al. (2007) with a dispersion of 0.3 dex (dashed black line) and the green line for galaxies at $0.2<z<0.7$ from Noeske et al. (2007b). The thick part of each line represents the correlation in the mass range explored by each study and the thin line is an extrapolation to lower masses.

(A color version of this figure is available in the online journal.)

of 0.16 dex in SFR, the present galaxies lie well above the 0.5 dex line. They represent extreme outliers to the relationship at $z \sim 2$, like the submillimeter galaxies (SMGs) but orders of magnitude smaller and less massive, because we probe stellar masses in the range $10^{7.5}<M^{\star}<10^{9.5} M_{\odot} \mathrm{yr}^{-1}$, much lower than Daddi et al. (2007). Compared to the $z \sim 1$ GOODS sample (Elbaz et al. 2007), for which the SFR $-M^{\star}$ relationship has 0.3 dex dispersion, the high-EW sample of galaxies is offset by more than 1 dex from the median value of SFR at a given mass. Noeske et al. (2007a) interpret $z \sim 1$ galaxies on the $\mathrm{SFR}-M^{\star}$ main sequence as the early phase of an SFH that smoothly declines for $\sim 1$ Gyr to $z \sim 0$. However, our observed sSFRs denote vigorous star formation episodes with very rapid stellar mass buildup, doubling total stellar mass in $<100 \mathrm{Myr}$. The observed offset of our galaxies from the SFR $-M^{\star}$ main sequence is instead consistent with strong burst episodes rather than a smoothly declining SFH.

\section{EXTREMELY LOW-METALLICITY GALAXIES}

It has long been known that lower luminosity galaxies tend also to have lower metallicities according to the luminosity-metallicity relationship, although with a large scatter (Tremonti et al. 2004; Salzer et al. 2005; Savaglio et al. 2005; Erb et al. 2006a). Extremely metal-poor galaxies (XMPGs) with oxygen abundance of $\log (\mathrm{O} / \mathrm{H})+12 \leqslant 7.65$ as defined by Kunth $\&$ Östlin (2000) are very rare (e.g., Morales-Luis et al. 2011). In the local universe, large EWs galaxies have been surveyed using broadband color selection (Brown et al. 2008). Cardamone et al. (2009) found compact star-forming galaxies in the SDSS, called "green peas," that have very blue $r-i$ colors because of their very strong [O III $] \lambda 5007$ emission line. Kakazu et al.

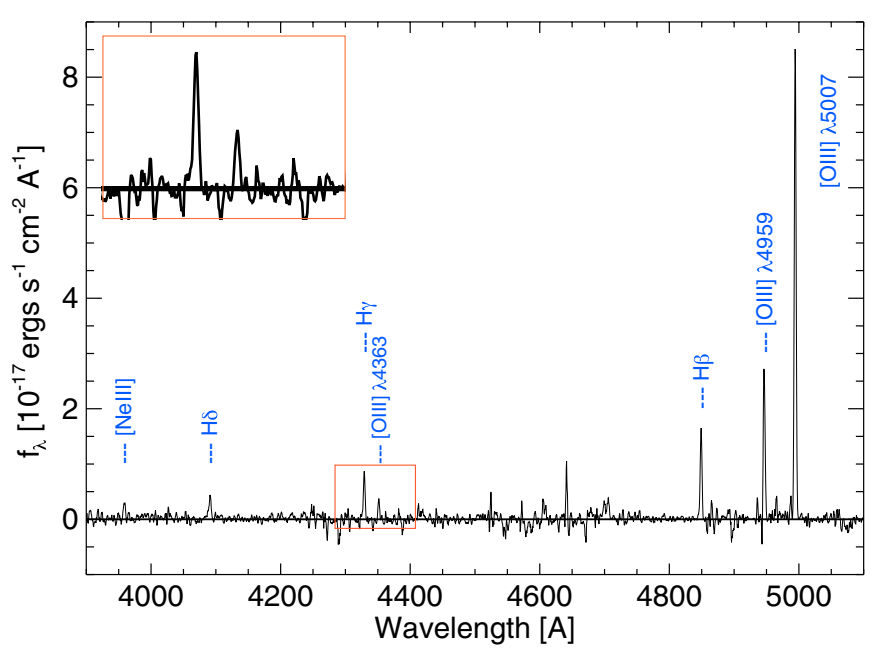

Figure 10. LRIS rest-frame spectrum of the XMPG WISP5_230 at $z \sim 0$.7. We observe a very faint continuum with very strong emission lines, such as $\mathrm{H} \beta$ or [O III] $\lambda 5007$. The inset shows a close-up of the $\mathrm{H} \gamma$ and [O III] $\lambda 4363$ lines. The detection of the [O III] $\lambda 4363$ auroral line suggests a very low metallicity. The spectrum has been smoothed by a 3 pixel filter.

(A color version of this figure is available in the online journal.)

(2007) showed that emission-line selection is yet more efficient than broadband selection and subsequent follow-up observations (e.g., DEEP2; Davis et al. 2003; Hoyos et al. 2005).

Many of the faint WISP galaxies have strong [O III] $\lambda 5007$ emission, and often weak [O II] $\lambda 3727$ emission, with a median ratio of $([\mathrm{O} \mathrm{III}] \lambda 5007 /[\mathrm{O} \mathrm{II}] \lambda 3727)=2.5$ (see also Hicks et al. 2002; Maier et al. 2006; Ly et al. 2007), suggestive of low metal abundances $(\log (\mathrm{O} / \mathrm{H}) \lesssim 8.5)$. We present here an example of a high-EW galaxy that shows a very low metallicity.

Amongst five high-EW galaxies for which we obtained LRIS spectra, only one galaxy was at a suitable redshift to observe the optical emission lines necessary for the metallicity measurement. The galaxy WISP5_230 proves to be an XMPG. It lies at a redshift of $z=0.7$ and its LRIS spectrum (Figure 10) confirms very strong emission lines seen in the grism spectra, with no detected continuum. In particular, the [O III] $\lambda 5007$ line has an EW lower limit of $\mathrm{EW}_{\text {rest }}>860 \AA$, and the $\mathrm{H} \beta$ line has $\mathrm{EW}_{\text {rest }}>180 \AA$. We also detect the [O III] $\lambda 4363$ auroral line, the presence of which always indicates a low metallicity, with $f\left(\left[\mathrm{O}_{\mathrm{III}}\right] \lambda 4363\right)=4.0 \pm 0.3 \times 10^{-18} \mathrm{erg} \mathrm{s}^{-1} \mathrm{~cm}^{-2}$, which is almost half of the flux of the $\mathrm{H} \gamma$ line. We used the direct method to derive the metallicity using the ratio of [O III] $\lambda \lambda 5007$, 4959 and $[\mathrm{O}$ III $] \lambda 4363$ that allows us to measure the electron temperature $t$. Using the NEBULAR package in IRAF (Shaw \& Dufour 1995), and assuming an electron density $n_{e}$ of $100 \mathrm{~cm}^{-3}$, we compute an electron temperature $t \sim 2 \times 10^{4} \mathrm{~K}$. We finally derive the oxygen abundance following the equations of Izotov et al. (2006):

$$
\begin{aligned}
12+\log \mathrm{O}^{+} / \mathrm{H}^{+}= & \log \frac{\lambda 3727}{\mathrm{H} \beta}+5.961+\frac{1.676}{t}-0.40 \log t \\
& -0.034 t+\log (1+1.35 x), \\
12+\log \mathrm{O}^{2+} / \mathrm{H}^{+}= & \log \frac{\lambda 4959+\lambda 5007}{\mathrm{H} \beta}+6.200+\frac{1.251}{t} \\
& -0.55 \log t-0.014 t,
\end{aligned}
$$

where $x=10^{-4} n_{e} t^{-0.5}$. Since the [O II] $\lambda 3727$ line fell on a sky line, we first use a fit to local H II galaxies used in Hoyos 


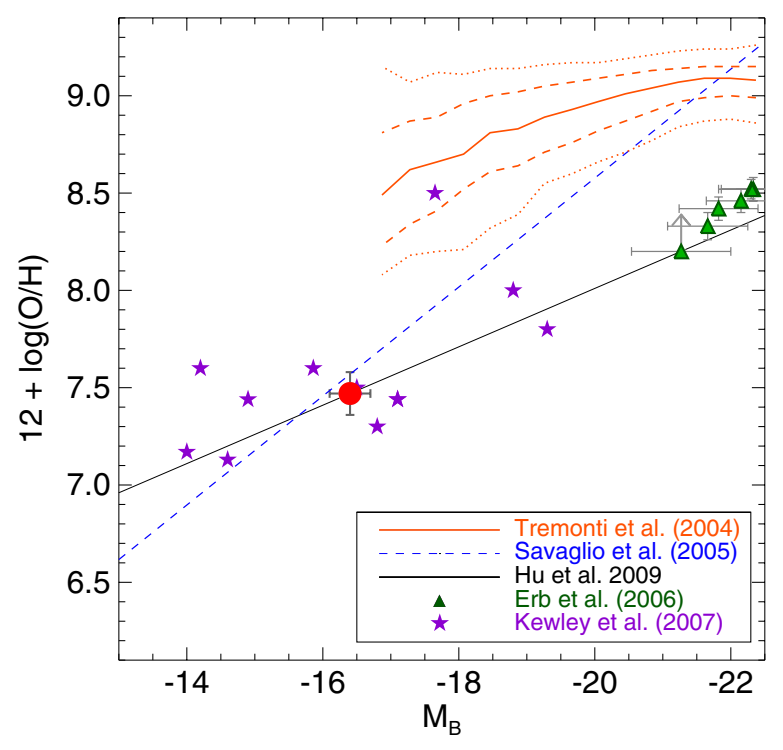

Figure 11. High-EW emission-line selection is uncovering extremely lowmetallicity objects. We show here the position of WISP5_230 in the metallicity-luminosity plane, together with results from the literature. The solid orange line represents the median of the low-redshift sample in SDSS of Tremonti et al. (2004), while the dashed and dotted orange lines are the contours that enclose $68 \%$ and $95 \%$ of the sample. The blue dashed line denotes the $z \sim 0.7$ galaxy sample of Savaglio et al. (2005) and the green triangles the $z \sim 2.3$ star-forming galaxies of Erb et al. (2006a) divided into six bins of luminosity. Local XMPGs and gamma-ray burst hosts from Kewley et al. (2007) are shown by magenta stars and XMPGs of the USEL sample are represented by the black solid line (Hu et al. 2009). The position of WISP5_230 is marked with a big red circle.

(A color version of this figure is available in the online journal.)

et al. (2005), which gives the ([O III $] \lambda 5007 /[\mathrm{O}$ II $] \lambda 3727)$ ratio as a function of $\mathrm{H} \beta \mathrm{EW}$, where the typical uncertainty on the [O II] flux is about $50 \%$. Then, using the typical ratio of 2.5 observed for the WISP galaxies we obtain a [O II] flux higher by $50 \%$ than the previous estimate. We eventually adopt the typical $[\mathrm{O} \mathrm{III}] /\left[\mathrm{O}_{\mathrm{II}}\right]$ line ratio observed in WISP as the final value. In fact, the uncertainties do not strongly depend on the precise value of the [O II] flux. The flux errors were propagated during the calculation. Finally, we obtain an oxygen abundance of $12+$ $\log (\mathrm{O} / \mathrm{H})=7.47 \pm 0.11$. For comparison, the solar metallicity of $Z=0.02$ translates to $12+\log (\mathrm{O} / \mathrm{H}) \sim 8.9$.

This object is comparable to the most metal-deficient galaxies found in the USEL sample (Hu et al. 2009) and close to the lowest metallicity star-forming galaxies known, IZw 18 (Thuan \& Izotov 2005) and SBS0335-52 (Izotov et al. 2006). We plot the metallicity and the absolute $B$-band magnitude of WISP5_230 in Figure 11 and compare it with results at different redshifts. The luminosity-metallicity relation is an important indicator of the chemical enrichment of the interstellar medium and the mass loss of galaxies. In the same figure, we show the relation established for a large sample of low-redshift star-forming galaxies in the SDSS (orange lines; Tremonti et al. 2004), at redshifts $0.4<z<1$ (blue dashed line; Savaglio et al. 2005), and for high-redshift LBGs at $z \sim 2.3$ (green triangles; Erb et al. 2006a). With an absolute magnitude of $M_{B} \sim-16.4$, WISP5_230 is comparable to local XMPGs (magenta stars), including the blue compact galaxy SDSS 0809+1729 found by Kewley et al. (2007).

We observe that, at constant luminosity, it has a metallicity $\sim 1$ dex lower than what would be predicted from local SDSS galaxies (Tremonti et al. 2004). It is better reproduced by the

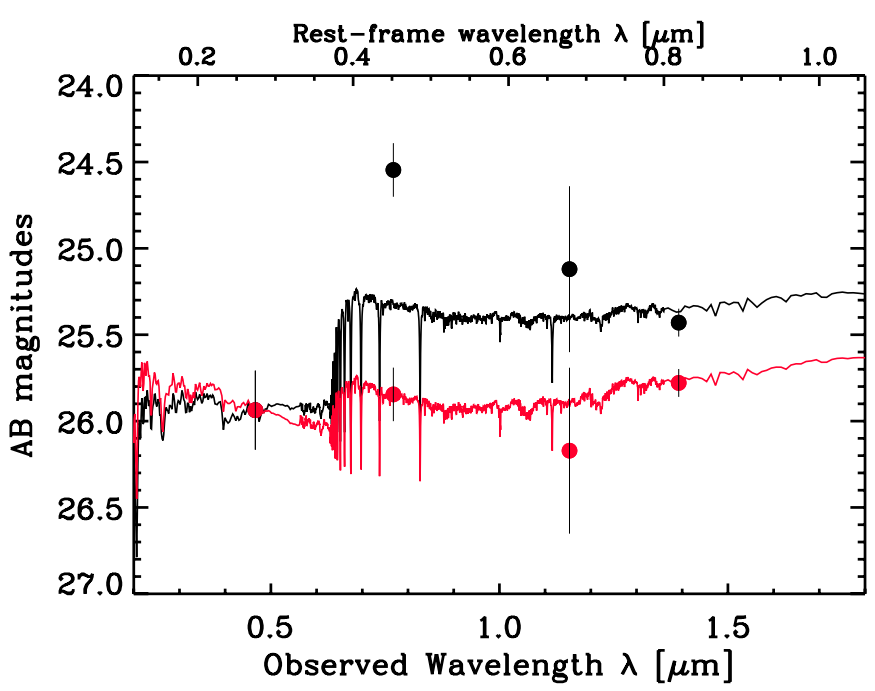

Figure 12. Spectral energy distribution fitting of XMPG WISP5_230. The black points represent the observed magnitudes in the $g^{\prime}, i^{\prime}, J$, and $H$ bands. The red points are the magnitudes corrected for the contribution of nebular emission lines. The $J$ and $H$ fluxes are corrected using the WFC3 grism spectra, and the $g^{\prime}$ flux using the LRIS spectrum. The solid black (red) curves is the best-fit SED to the raw (emission-line corrected) magnitudes using Bruzual \& Charlot (2003) models. The SED fitting procedure is detailed in Section 5.2.

(A color version of this figure is available in the online journal.)

black solid line in Figure 11 that represents the same trend for the USEL sample (Hu et al. 2009). It is also consistent with the relationship of Savaglio et al. (2005) derived for $z \sim 0.7$ galaxies.

This object also has a stellar mass of $\log \left(M^{\star}\right)=7.67 \pm$ $0.13 M_{\odot}$, the lowest value in the sample of nine galaxies for which we performed SED modeling (see Section 5.2). The best-fit SED is shown in Figure 12. The low metallicity and low mass of this object are consistent with the well-known mass-metallicity relation (Tremonti et al. 2004; Savaglio et al. 2005; Erb et al. 2006a; Mannucci et al. 2009). In addition, Mannucci et al. (2010) argue that the mass-metallicity relation is simply a projection of a more fundamental relation between mass, gas-phase metallicity, and SFR. The evolution of the mass-metallicity relation up to $z=2.5$ would be the result of an evolution within this fundamental relation, as higher-SFR galaxies are being selected at increasing redshifts. Therefore, for low-mass galaxies, the metallicity decreases sharply with increasing SFR (see also Lara-Lopez et al. 2010). However, the high-redshift samples are still small and there is not enough overlap between SFRs of the low- and high- $z$ samples to confirm this scenario. Using the extension of the fundamental metallicity equation down to $\log (M) \sim 7.8$ published in Mannucci et al. (2011) we find that, with $\mathrm{SFR} \sim 1.9 M_{\odot} \mathrm{yr}^{-1}$, the galaxy presented here would have an oxygen abundance of about 12 $+\log (\mathrm{O} / \mathrm{H}) \sim 7.7 \pm 0.4$. It appears that this galaxy fits in a scenario of no evolution in the $M^{\star}-Z-S F R$ fundamental plane. A large sample of galaxies is needed to test this scenario, and this will be the subject of a forthcoming paper (A. Henry et al. 2011 , in preparation).

\section{SUMMARY}

The WISP Survey (Atek et al. 2010) offers a unique opportunity to search for high-redshift strong emission-line galaxies, down to a line flux limit of $\sim 5 \times 10^{-17} \mathrm{erg} \mathrm{s} \mathrm{cm}^{-1}$, regardless of their continuum brightness, thanks to unprecedented 
IR grism capabilities of the new WFC3 instrument onboard the HST.

We selected in this work a sample of 176 objects in 54 fields, with rest-frame EW higher than $200 \AA$, spanning a redshift range of $0.35<z<2.3$. This selection results in a surface density of one object per square arcmin.

The presence of such strong emission lines in the spectra of star-forming galaxies at $0.8<z<2.35$ has important implications for the study of high-redshift galaxy population. We first show that the contribution of nebular lines to the total broadband flux density can be more than $1 \mathrm{mag}$, with a median value at $0.3 \mathrm{mag}$. We demonstrate that strong emission lines falling in the IR broadband filters can mimic the $Y_{098}-J_{125}$ and $J_{125}-H_{160}$ color criteria used to select $z \sim 8$ galaxy candidates. While the presence of such interlopers in deep HUDF observations is unlikely, the wide WFC3 pure parallel surveys are prone to such contamination because of the lack of deep optical observations.

High-EW emission lines can significantly affect the SED modeling of high- $z$ galaxies and consequently the derived physical properties. We show that, when the emission lines create the appearance of a Balmer/4000 $\AA$ break, the age and mass can be overestimated by an average factor of 2 and up to a factor of 10. More importantly, the situation will be much more problematic at higher redshift where we expect higher EWs. Our results confirm empirically the theoretical predictions of Schaerer \& de Barros (2009) in a large sample of galaxies and call for a careful treatment of nebular lines in SED fitting of star-forming galaxies at all redshifts.

The high-EW sample consists of young, unevolved galaxies with high sSFRs, which appear to be extreme outliers of the main sequence of the SFR $-M^{\star}$ relationship. The high-EW selection can sample metal-deficient galaxies. As an example, we obtained rest-frame optical spectroscopy with LRIS at Keck for five of these objects, but only one of them was at the right redshift for the metallicity measurement. This galaxy is located at $z \sim 0.7$ and is an XMPG. It follows the metallicity-luminosity relation derived from the USEL sample (Hu et al. 2009) but is also consistent with $z \sim 0.7$ galaxies (Savaglio et al. 2005). With an oxygen abundance of $12+\log (\mathrm{O} / \mathrm{H})=7.47 \pm 0.11$, it is amongst the lowest metallicities in the samples of XMPGs (Kunth \& Östlin 2000; Kakazu et al. 2007; Kewley et al. 2007). A larger sample of low-metallicity galaxies will be analyzed in a forthcoming paper (A. Henry et al. 2011, in preparation), but we already demonstrate that we can find faint metal-poor galaxies in the high-EW sample to be targeted in follow-up spectroscopy.

We thank the anonymous referee for very useful comments that improved the content and the clarity of the paper, and Ranga-Ram Chary and Yuko Kakazu for interesting discussions. We thank Martin Kümmel, Harald Kuntschner, Jeremy Walsh, Howard Bushouse, and the staff members of the Space Telescope Institute for their help with the data reduction. We also wish to recognize and acknowledge the very significant cultural role and reverence that the summit of Mauna Kea has always had within the indigenous Hawaiian community. We are most fortunate to have the opportunity to conduct observations from this mountain.

\section{REFERENCES}

Atek, H., Kunth, D., Schaerer, D., et al. 2009, A\&A, 506, L1 Atek, H., Malkan, M., McCarthy, P., et al. 2010, ApJ, 723, 104 Bouché, N., Dekel, A., Genzel, R., et al. 2010, ApJ, 718, 1001
Bouwens, R. J., Illingworth, G. D., Labbe, I., et al. 2011, Nature, 469, 504 Bouwens, R. J., Illingworth, G. D., Oesch, P. A., et al. 2011b, ApJ, 737, 90 Brinchmann, J., Charlot, S., White, S. D. M., et al. 2004, MNRAS, 351, 1151 Brinchmann, J., \& Ellis, R. S. 2000, ApJ, 536, L77

Brinchmann, J., Pettini, M., \& Charlot, S. 2008, MNRAS, 385, 769

Brown, W. R., Kewley, L. J., \& Geller, M. J. 2008, AJ, 135, 92

Bruzual, G., \& Charlot, S. 2003, MNRAS, 344, 1000

Bunker, A. J., Wilkins, S., Ellis, R. S., et al. 2010, MNRAS, 409, 855

Cardamone, C., Schawinski, K., Sarzi, M., et al. 2009, MNRAS, 399, 1191

Chabrier, G. 2003, ApJ, 586, L133

Cowie, L. L., Barger, A. J., \& Hu, E. M. 2010, ApJ, 711, 928

Cowie, L. L., Barger, A. J., \& Hu, E. M. 2011, ApJ, 738, 136

Daddi, E., Cimatti, A., Renzini, A., et al. 2004, ApJ, 617, 746

Daddi, E., Dickinson, M., Morrison, G., et al. 2007, ApJ, 670, 156

Damen, M., Labbé, I., Franx, M., et al. 2009, ApJ, 690, 937

Davé, R., Oppenheimer, B. D., \& Finlator, K. 2011, MNRAS, 415, 11

Davis, M., Faber, S. M., Newman, J., et al. 2003, Proc. SPIE, 4834, 161

Elbaz, D., Daddi, E., Le Borgne, D., et al. 2007, A\&A, 468, 33

Erb, D. K., Shapley, A. E., Pettini, M., et al. 2006a, ApJ, 644, 813

Erb, D. K., Steidel, C. C., Shapley, A. E., et al. 2006b, ApJ, 647, 128

Eyles, L. P., Bunker, A. J., Ellis, R. S., et al. 2007, MNRAS, 374, 910

Finkelstein, S. L., Hill, G. J., Gebhardt, K., et al. 2011, ApJ, 729, 140

Finkelstein, S. L., Papovich, C., Giavalisco, M., et al. 2010, ApJ, 719, 1250

Finlator, K., Oppenheimer, B. D., \& Davé, R. 2011, MNRAS, 410, 1703

Grogin, N. A., Kocevski, D. D., Faber, S. M., et al. 2011, arXiv:1105.3753

Hao, L., Strauss, M. A., Tremonti, C. A., et al. 2005, AJ, 129, 1783

Hathi, N. P., Ryan, R. E., Jr., Cohen, S. H., et al. 2010, ApJ, 720, 1708

Hicks, E. K. S., Malkan, M. A., Teplitz, H. I., McCarthy, P. J., \& Yan, L. 2002, ApJ, 581, 205

Hopkins, A. M. 2004, ApJ, 615, 209

Hoyos, C., Koo, D. C., Phillips, A. C., Willmer, C. N. A., \& Guhathakurta, P. 2005, ApJ, 635, L21

Hu, E. M., Cowie, L. L., Kakazu, Y., \& Barger, A. J. 2009, ApJ, 698, 2014

Inoue, A. K. 2011, MNRAS, 415, 2920

Izotov, Y. I., Stasińska, G., Meynet, G., Guseva, N. G., \& Thuan, T. X. 2006, A\&A, 448, 955

Izotov, Y. I., \& Thuan, T. X. 2008, ApJ, 687, 133

Jester, S., Schneider, D. P., Richards, G. T., et al. 2005, AJ, 130, 873

Kakazu, Y., Cowie, L. L., \& Hu, E. M. 2007, ApJ, 668, 853

Kashikawa, N., Shimasuku, K., Matsuda, Y., et al. 2011, ApJ, 734, 119

Kennicutt, R. C., Jr. 1998, ARA\&A, 36, 189

Kewley, L. J., Brown, W. R., Geller, M. J., Kenyon, S. J., \& Kurtz, M. J. 2007, AJ, 133,882

Kewley, L. J., Dopita, M. A., Sutherland, R. S., Heisler, C. A., \& Trevena, J. 2001, ApJ, 556, 121

Kewley, L. J., Groves, B., Kauffmann, G., \& Heckman, T. 2006, MNRAS, 372 , 961

Kimble, R. A., MacKenty, J. W., O’Connell, R. W., \& Townsend, J. A. 2008, Proc. SPIE, 7010, 43

Kriek, M., van Dokkum, P. G., Labbé, I., et al. 2009, ApJ, 700, 221

Kümmel, M., Walsh, J. R., Pirzkal, N., Kuntschner, H., \& Pasquali, A. 2009, PASP, 121, 59

Kunth, D., \& Östlin, G. 2000, A\&AR, 10, 1

Labbé, I., González, V., Bouwens, R. J., et al. 2010, ApJ, 716, L103

Lara-Lopez, M., Cepa, J., Bongiovanni, A., et al. 2010, A\&A, 521L, 53L

Leitherer, C., Schaerer, D., Goldader, J. D., et al. 1999, ApJS, 123, 3

Lorenzoni, S., Bunker, A. J., Wilkins, S. M., et al. 2011, MNRAS, 414, 1455

Ly, C., Malkan, M. A., Kashikawa, N., et al. 2007, ApJ, 657, 738

Maier, C., Lilly, S. J., Carollo, C. M., et al. 2006, ApJ, 639, 858

Mannucci, F., Cresci, G., Maiolino, R., et al. 2009, MNRAS, 398, 1915

Mannucci, F., Cresci, G., Maiolino, R., Marconi, A., \& Gnerucci, A. 2010, MNRAS, 408, 2115

Mannucci, F., Salvaterra, R., \& Campisi, M. A. 2011, arXiv:1011.4506

McLinden, E. M., Finkelstein, S. L., Rhoads, J. E., et al. 2011, ApJ, 730, 136

McLure, R. J., Dunlop, J. S., Cirasuolo, M., et al. 2010, MNRAS, 403, 960

McLure, R. J., Dunlop, J. S., de Rovel, L., et al. 2011, arXiv:1102.4881

Morales-Luis, A. B., Sanchez Almeida, J., Aguerri, J. A. L., \& Munoz-Tunon, C. 2011, arXiv:1109.0235

Nilsson, K. K., Tapken, C., Møller, P., et al. 2009, A\&A, 498, 13

Noeske, K. G., Faber, S. M., Weiner, B. J., et al. 2007a, ApJ, 660, L47

Noeske, K. G., Weiner, B. J., Faber, S. M., et al. 2007b, ApJ, 660, L43

Oesch, P. A., Bouwens, R. J., Illingworth, G. D., et al. 2010, ApJ, 709, L16

Oke, J. B., Cohen, J. G., Carr, M., et al. 1995, PASP, 107, 375

Oliver, S., Frost, M., Farrah, D., et al. 2010, MNRAS, 405, 2279 
Ono, Y., Ouchi, M., Shimasaku, K., et al. 2010, ApJ, 724, 1524

Osterman, S., Green, J., Froning, C., et al. 2011, Ap\&SS, 335, 257

Ouchi, M., Shimasaku, K., Akiyama, M., et al. 2008, ApJS, 176, 301

Ouchi, M., Shimasaku, K., Furusawa, H., et al. 2010, ApJ, 723, 869

Pannella, M., Carilli, C. L., Daddi, E., et al. 2009, ApJ, 698, L116

Papovich, C., Moustakas, L. A., Dickinson, M., et al. 2006, ApJ, 640, 92

Raiter, A., Fosbury, R. A. E., \& Teimoorinia, H. 2010, A\&A, 510, A109

Salzer, J. J., Lee, J. C., Melbourne, J., et al. 2005, ApJ, 624, 661

Sargent, M. T., Carollo, C. M., Lilly, S. J., et al. 2007, ApJS, 172, 434

Savaglio, S., Glazebrook, K., Le Borgne, D., et al. 2005, ApJ, 635, 260

Scalo, J. M. 1986, Fund. Cosm. Phys., 11, 1

Scarlata, C., Colbert, J., Teplitz, H. I., et al. 2009, ApJ, 704, L98

Schaerer, D., \& de Barros, S. 2009, A\&A, 502, 423

Schiminovich, D., Wyder, T. K., Martin, D. C., et al. 2007, ApJS, 173, 315

Shapley, A. E., Steidel, C. C., Erb, D. K., et al. 2005, ApJ, 626, 698

Shapley, A. E., Steidel, C. C., Pettini, M., \& Adelberger, K. L. 2003, ApJ, 588, 65
Shaw, R. A., \& Dufour, R. J. 1995, PASP, 107, 896

Shim, H., Chary, R.-R., Dickinson, M., et al. 2011, ApJ, 738, 69

Steidel, C. C., Giavalisco, M., Dickinson, M., \& Adelberger, K. L. 1996, AJ, 112,352

Steidel, C. C., Shapley, A. E., Pettini, M., et al. 2004, ApJ, 604, 534

Taniguchi, Y., Shioya, Y., \& Trump, J. R. 2010, ApJ, 724, 1480

Thuan, T. X., \& Izotov, Y. I. 2005, ApJS, 161, 240

Tremonti, C. A., Heckman, T. M., Kauffmann, G., et al. 2004, ApJ, 613, 898

Trenti, M., Bradley, L. D., Stiavelli, M., et al. 2011, ApJ, 727, L39

Vanzella, E., Giavalisco, M., Dickinson, M., et al. 2009, ApJ, 695, 1163

Veilleux, S., \& Osterbrock, D. E. 1987, ApJS, 63, 295

Watson, D., French, J., Christensen, L., et al. 2010, arXiv:1010.1783

Woodgate, B. E., Kimble, R. A., Bowers, C. W., et al. 1998, PASP, 110, 1183

Yan, H., Windhorst, R. A., Hathi, N. P., et al. 2010, Res. Astron. Astrophys., 10, 867

Yan, H., Yan, L., Zamojski, M. A., et al. 2011, ApJ, 728, L22

York, D. G., Adelman, J., Anderson, J. E., Jr., et al. 2000, AJ, 120, 1579 